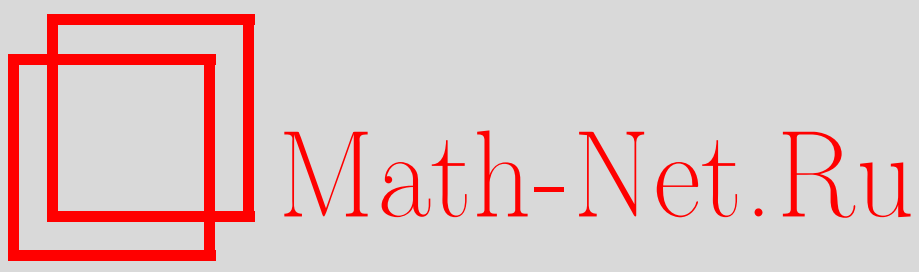

В. В. Зудилин, Об оценках снизу многочленов от значений некоторых целых функций, Матем. сб., 1996, том 187, номер 12, 57-86

DOI: https://doi.org/10.4213/sm178

Использование Общероссийского математического портала Math-Net.Ru подразумевает, что вы прочитали и согласны с пользовательским соглашением

http: //www. mathnet.ru/rus/agreement

Параметры загрузки:

IP: 54.224 .187 .69

26 апреля 2023 г., $11: 55: 52$ 
УДК 511.36

\section{В. В. Зудилин}

\section{Об оценках снизу многочленов от значений некоторых целых функций}

В работе установлены оценки снизу многочленов и линейных форм от значений $E$-функций в рациональной точке, зависящие от всех коэффициентов. Приведены следствия для обобщенных гипергеометрических $E$-фуункций.

Библиография: 9 названий.

\section{Введение}

История вопроса. Важным направлением теории диофантовых приближений и трансцендентных чисел является исследование поведения величины

$$
\left|h_{1} \xi_{1}+\cdots+h_{m} \xi_{m}\right|, \quad h_{j} \in \mathbb{Z}, \quad j=1, \ldots, m
$$

для заданных действительных $\xi_{1}, \ldots, \xi_{m}, m \geqslant 1$, и ее оценка снизу в зависимости от целых $h_{1}, \ldots, h_{m}$. Как следует из теоремы Дирихле (см., например, [1, гл. 1, $\S 2$, теорема 4]) для любого действительного $H \geqslant 1$ существуют иелье числа $h_{1}, \ldots, h_{m}$ такие, что

$$
\left|h_{1} \xi_{1}+\cdots+h_{m} \xi_{m}\right|<H^{-m+1}, \quad 0<\max _{1 \leqslant j \leqslant m}\left\{\left|h_{j}\right|\right\} \leqslant H
$$

Тем самым, теорема Дирихле отвечает на вопрос, сколь мальм может быть величина (0.1) в зависимости от

$$
\max _{1 \leqslant j \leqslant m}\left\{\left|h_{j}\right|\right\}
$$

При этом абсолютно не учитьвается структура чисел $\xi_{1}, \ldots, \xi_{m}$. Из метрических соображений [2, гл.I, теорема 12] следует, что при любом $\varepsilon>0$ для почти всех (в смысле меры Лебега) точек $\bar{\xi}=\left(\xi_{1}, \ldots, \xi_{m}\right) \in \mathbb{R}^{m}$ существует постоянная $C=C(\bar{\xi}, \varepsilon)>0$ такая, что для произвольных иельх $h_{1}, \ldots, h_{m}$, не равных нулю одновременно, справедливо неравенство

$$
\left|h_{1} \xi_{1}+\cdots+h_{m} \xi_{m}\right|>C\left(H_{1} \cdots H_{m}\right)^{-1} H(\log H)^{-m+1-\varepsilon}
$$

¿de

$$
H_{j}=\max \left\{1,\left|h_{j}\right|\right\}, \quad j=1, \ldots, m, \quad H=\max \left\{e, \max _{1 \leqslant j \leqslant m}\left\{H_{j}\right\}\right\} .
$$

Работа вьполнена при частичной поддержке Российского фонда фундаментальных исследований (грант № 97-01-00181).

(C) В. В. Зудилин 1996 
Однако, в настояшее время не известно ни одного набора чисел $\bar{\xi}$, для которого выполнялось бы такое неравенство.

Методы теории трансцендентных чисел позволяют при специальном выборе $\bar{\xi}$ получать оценки снизу величины (0.1) вида

$$
\left|h_{1} \xi_{1}+\cdots+h_{m} \xi_{m}\right|>C H^{-m+1-\varepsilon} .
$$

В то же время, в ряде работ, связанных, например, с оценками отклонения равномерно распределенных последовательностей, возникает необходимость в оценках снизу модулей линейных форм (0.1), причем оценках, зависяших от всех коэффициентов. Впервые, по всей видимости, подобньй результат был получен А. Бейкером для значений экспоненты [3]:

$$
\left|h_{1} e^{\alpha_{1}}+\cdots+h_{m} e^{\alpha_{m}}\right|>C\left(H_{1} \cdots H_{m}\right) H^{1-\gamma / \sqrt{\log \log H}} .
$$

Для этих целей он несколько уточнил метод, предложенный К. Л. Зигелем [4], но серьезного обобшения схема Бейкера так и не получила (попытка сделать это содержится в работе [5]).

Задача оценки снизу линейных форм от действительных чисел может быть естественным образом обобщена. Именно, можно исследовать поведение величины

$$
\left|P\left(\xi_{1}, \ldots, \xi_{m}\right)\right|, \quad P \in \mathbb{Z}\left[y_{1}, \ldots, y_{m}\right],
$$

в зависимости от коэффициентов многочлена $P\left(y_{1}, \ldots, y_{m}\right)$ (например, его высоты $H=H(P))$ и его степени $d=\operatorname{deg} P$.

Упомянутый выше метод Зигеля позволяет проводить эти исследования в случае, когда в качестве чисел $\xi_{1}, \ldots, \xi_{m}$ рассматриваются значения в алгебраической точке $\alpha \in \mathbb{K} \backslash\{0\}$ аналитических функций

$$
f_{j}(z)=\sum_{\nu=0}^{\infty} f_{j, \nu} z^{\nu}, \quad f_{j, \nu} \in \mathbb{K}, \quad j=1, \ldots, m, \quad \nu \in \mathbb{Z}^{+}=\{0,1,2, \ldots\},
$$

составляюших в совокупности решение системы линейных дифференциальных уравнений

$$
\begin{gathered}
\frac{d}{d z} y_{l}=\sum_{j=1}^{m} Q_{l j} y_{j}, \quad l=1, \ldots, m, \\
Q_{l j}=Q_{l j}(z) \in \mathbb{C}(z), \quad l, j=1, \ldots, m,
\end{gathered}
$$

первого порядка. При этом коэффициенты в рядах Тейлора функций (0.3) удовлетворяют некоторьм дополнительньм арифметическим условиям, определяюшим класс $E$-функций, и точка $\alpha$ не является особой точкой системы (0.4). Метод Зигеля был сушественно усилен в работах А.Б. Шидловского, который, в частности, доказал критерий алгебраической независимости значений $E$-функций. Подробную историю этого вопроса можно найти в его монографии [1]. Сразу отметим, что в дальнейшем будет рассматриваться только случай $\mathbb{K}=\mathbb{Q}$, поскольку все оценки, полученные в нем, имеют свои естественные аналоги для произвольного конечного расширения поля рациональных чисел. 
ОПРЕДЕЛЕНИЕ [6]. Функция

$$
f(z)=\sum_{\nu=0}^{\infty} \frac{f_{\nu}}{\nu !} z^{\nu}, \quad f_{\nu} \in \mathbb{Q}, \quad \nu \in \mathbb{Z}^{+}
$$

называется $E$-функиией, если для некоторой положительной константы $C$ справедливо неравенство $\left|f_{\nu}\right|<C^{\nu+1}$ при $\nu \in \mathbb{Z}^{+}$и сушествует последовательность натуральных чисел $\left\{\varphi_{n}\right\}_{n=1}^{\infty}$ такая, что $\varphi_{n} f_{\nu} \in \mathbb{Z}, \nu=0,1, \ldots, n, n \in \mathbb{N}$, и $\varphi_{n}<C^{n}$ при $n \in \mathbb{N}$.

Данное определениенесколько отличаетсяот классического определенияЗигеля. Однако, все известные $E$-функции (в смысле Зигеля) с рациональными коэффициентами рядов Тейлора, являюшиеся решениями линейных дифференциальных уравнений, удовлетворяют этому определению. В частности, это относится ко всем целым гипергеометрическим функциям с рациональньми параметрами (см. $[1$, гл. $5, \S 1])$.

В 1984 году Г.В. Чудновский [7] предложил оригинальную конструкцию, позволяющую получать оценки снизу линейных форм от значений $E$-функций $(0.3)$, каждая из которых удовлетворяет своему линейному однородному дифференциальному уравнению произвольного порядка:

$$
\left|h_{1} f_{1}(\alpha)+\cdots+h_{m} f_{m}(\alpha)\right|>C\left(H_{1} \cdots H_{m}\right)^{-1} H^{1-\varepsilon} .
$$

При этом на совокупность этих уравнений накладывалось очень жесткое ограничительное условие, схожее с условием Зигеля. Кроме этого, Чудновскому не удалось корректно осуществить переход от линейных приближающих функциональных форм, названных им градуированными приближениями Паде, к линейньм числовым формам. В целом, метод Чудновского являлся непосредственным развитием метода Зигеля-Шидловского. Дальнейшая реализация конструкции градуированных приближений Паде в работе [8] привела к доказательству точных по порядку оценок меры иррациональности значений $E$-функций (0.3):

$$
\left|f_{l}(\alpha)-\frac{p}{q}\right|>|q|^{-2-\gamma(\log \log |q|)^{-1 /(m+1)}}, \quad l=1, \ldots, m,
$$

с легко проверяемьм условием на совокупность рассматриваемых функций.

В настоящей работе с помощью новых идей в методе Зигеля-Шидловского конструкция градуированных приближений Паде реализована в полном объеме для получения оценок снизу величин (0.1) и (0.2). При этом условие, накладываемое на совокупность рассматриваемых функций, является более простым по сравнению с условием Чудновского и во многих случаях проверено. Это позволяет получить следствия для конкретных обобщенных гипергеометрических функций.

Основные результаты. Будем говорить, что система линейных однородных дифференциальных уравнений первого порядка (0.4) лежит в клас$c e \mathbf{W}^{0}$, если функции, входящие в некоторую фундаментальную матрицу решений $\left(\psi_{j l}\right)_{j, l=1, \ldots, m}$ системы $(0.4)$, однородно алгебраически независимы над $\mathbb{C}(z)$. Отметим, что в этом случае "некоторую фундаментальную матрицу решений" можно заменить на "произвольную фундаментальную матрицу решений", поскольку все такие матрицы отличаются матричньм множителем с постоянными коэффициентами. 
ТеОРема I. Пусть совокупность Е-функиий $f_{1}(z), \ldots, f_{m}(z), m \geqslant 2$, удовлетворяет системе линейных однородных дифференциальных уравнений (0.4) из класса $\mathbf{W}^{0}, \alpha \in \mathbb{Q} \backslash\{0\}$ - неособая точка этой системы и $d \in \mathbb{N}$. Тогда существуют полохсительные постоянные $\gamma=\gamma\left(f_{1}, \ldots, f_{m} ; \alpha, d\right)$ u $C=C\left(f_{1}, \ldots, f_{m}\right.$; $\alpha, d)$ такие, что для любого однородного многочлена $P \in \mathbb{Z}\left[y_{1}, \ldots, y_{m}\right]$ степени d выполняется неравенство

$$
\begin{gathered}
\left|P\left(f_{1}(\alpha), \ldots, f_{m}(\alpha)\right)\right|>C \cdot\left|h_{1} \cdots h_{w}\right|^{-1} H^{1-\gamma(\log \log H)^{-1 /\left(m^{2}-m+2\right)},} \\
H=\max _{1 \leqslant i \leqslant w}\left\{\left|h_{i}\right|\right\} \geqslant 3,
\end{gathered}
$$

где $h_{1}, \ldots, h_{w}$ - все ненулевъе коэффициенты многочлена $P\left(y_{1}, \ldots, y_{m}\right)$.

Из теоремы I непосредственно вытекает результат об оценке снизу линейных форм от значений $E$-функций.

СЛЕДСТВИЕ. Пусть совокупность Е-функиий $f_{1}(z), \ldots, f_{m}(z), m \geqslant 2$, удовлетворяет системе линейных однородных дифференииальных уравнений (0.4) из класса $\mathbf{W}^{0}, \alpha \in \mathbb{Q} \backslash\{0\}$ - неособая точка этой системы. Тогда существуют полохсительные постоянные $\gamma=\gamma\left(f_{1}, \ldots, f_{m} ; \alpha\right) u C=C\left(f_{1}, \ldots, f_{m} ; \alpha\right)$ такие, что

$$
\begin{gathered}
\left|h_{1} f_{1}(\alpha)+\cdots+h_{m} f_{m}(\alpha)\right|>C \cdot\left(H_{1} \cdots H_{m}\right)^{-1} H^{1-\gamma(\log \log H)^{-1 /\left(m^{2}-m+2\right)}}, \\
h_{i} \in \mathbb{Z}, \quad H_{i}=\max \left\{1,\left|h_{i}\right|\right\}, \quad i=1, \ldots, m, \quad H=\max _{1 \leqslant i \leqslant m}\left\{H_{i}\right\} \geqslant 3 .
\end{gathered}
$$

На самом деле, теорема I является частным случаем некоторого более общего утверждения, которое и будет доказываться ниже. Для его формулировки нам понадобится дополнительное понятие. В дальнейшем будет рассматриваться система линейных однородных дифференциальных уравнений первого порядка, распадаюшаяся на $m$ подсистем:

$$
\begin{gathered}
\frac{d}{d z} y_{i l}=\sum_{j=1}^{m_{i}} Q_{l j}^{(i)} y_{i j}, \quad l=1, \ldots, m_{i}, \\
Q_{l j}^{(i)}=Q_{l j}^{(i)}(z) \in \mathbb{Q}(z), \quad l=1, \ldots, m, \quad m \geqslant 2 .
\end{gathered}
$$

Если $\alpha \in \mathbb{C}$ - неособая точка системы (0.5), то она является неособой точкой и для системы линейных однородных дифференциальных уравнений

$$
\frac{d}{d z} a_{i j}=-\sum_{l=1}^{m_{i}} Q_{l j}^{(i)} a_{i l}, \quad j=1, \ldots, m_{i}, \quad i=1, \ldots, m,
$$

сопряженной к системе (0.5). Поэтому сушествует набор аналитических в некоторой окрестности точки $z=\alpha$ функций

$$
\varphi_{i j}=\varphi_{i j}(z), \quad j=1, \ldots, m_{i}, \quad i=1, \ldots, m,
$$

удовлетворяющих системе (0.6), такой, что

$$
\varphi_{i j}(\alpha)=\left\{\begin{array}{l}
1 \text { при } j=1, \\
0 \text { при } j=2, \ldots, m_{i},
\end{array} \quad i=1, \ldots, m .\right.
$$

Если функции (0.7) однородно алгебраически независимы над $\mathbb{C}(z)$, то будем говорить, что система (0.5) принадлежит классу $\mathbf{W}^{0}(\alpha)$. 
Tеорема II. Пусть совокупность Е-функций

$$
f_{i l}(z), \quad l=1, \ldots, m_{i}, \quad i=1, \ldots, m,
$$

удовлетворяет системе линейных однородных дифференциальных уравнений $(0.5)$ из класса $\mathbf{W}^{0}(\alpha)$. Для прочзвольного однородного многочлена $P=P\left(y_{1}, \ldots, y_{m}\right) \in \mathbb{Z}\left[y_{1}, \ldots, y_{m}\right]$ степени $d \in \mathbb{N}$ введем множество мультииндексов $U$ таким образом, ито

$$
\begin{gathered}
P\left(y_{1}, \ldots, y_{m}\right)=\sum_{\bar{u} \in U} h_{\bar{u}} y_{1}^{u_{1}} \cdots y_{m}^{u_{m}}, \\
h_{\bar{u}} \neq 0, \quad|\bar{u}|=u_{1}+\cdots+u_{m}=d, \quad \bar{u} \in U ;
\end{gathered}
$$

при этом функции

$$
F_{\bar{u}}(z)=f_{11}^{u_{1}}(z) f_{21}^{u_{2}}(z) \cdots f_{m 1}^{u_{m}}(z), \quad \bar{u} \in U
$$

линейно независимы над $\mathbb{C}(z)$. Тогда существуют положительные постоянные $\gamma$ и $C$, зависящие только от совокупности функиий (0.9), числа $d u$ неособой точки $\alpha \in \mathbb{Q} \backslash\{0\}$, такие, что справедливо неравенство

$$
\begin{aligned}
& \left|P\left(f_{11}(\alpha), f_{21}(\alpha), \ldots, f_{m 1}(\alpha)\right)\right|>C \prod_{\bar{u} \in U}\left|h_{\bar{u}}\right|^{-1} H^{1-\gamma(\log \log H)^{-1 /\left(m_{1}+\cdots+m_{m}-m+2\right)},} \\
& H=\max _{\bar{u} \in U}\left\{\left|h_{\bar{u}}\right|\right\} \geqslant 3
\end{aligned}
$$

ДОКАЗАТЕЛЬСТВО ТЕОРЕМЫ І. Для совокупности функций $f_{1}(z), \ldots, f_{m}(z)$ из теоремы I рассмотрим $m$ "роллированых" копий

$$
f_{i l}(z) \equiv\left\{\begin{array}{ll}
f_{i-l+1}(z), & \text { если } i-l \geqslant 0, \\
f_{i-l+1+m}(z), & \text { если } i-l<0,
\end{array} \quad i, l=1, \ldots, m,\right.
$$

каждая из которых удовлетворяет системе линейных однородных дифференциальных уравнений, полученной из системы (0.4) некоторой перестановкой индексов. Отметим сразу, что поскольку исходные функции $f_{1}(z), \ldots, f_{m}(z)$, входят в некоторую фундаментальную систему решений системы $(0.4)$ из класса $\mathbf{W}^{0}$, они однородно алгебраически независимы над $C(z)$.

Пусть

$$
\psi_{l j}(z), \quad l, j=1, \ldots, m,
$$

- элементы фундаментальной матрицы решений системы (0.4), преврашаюшейся в единичную матрицу в точке $z=\alpha$ :

$$
\psi_{l j}(\alpha)=\delta_{l j}, \quad l, j=1, \ldots, m .
$$


При этом функции (0.12) однородно алгебраически независимы над $\mathbb{C}(z)$, поскольку система (0.4) лежит в классе $\mathbf{W}^{0}$. Тогда элементы обратной матрицы

$$
\left(\widetilde{\psi}_{l j}(z)\right)_{l, j=1, \ldots, m}=\left(\psi_{l j}(z)\right)_{l, j=1, \ldots, m}^{-1},
$$

как рациональные функции от (0.12), также однородно алгебраически независимы над $\mathbb{C}(z)$, и, кроме того, согласно $(0.13)$ для них вьполнены условия нормировки в точке $z=\alpha$ :

$$
\widetilde{\psi}_{l j}(\alpha)=\delta_{l j}, \quad l, j=1, \ldots, m .
$$

Остается заметить, что однородно алгебраически независимые над $\mathbb{C}(z)$ функции

$$
\varphi_{i j}(z)=\left\{\begin{array}{ll}
\widetilde{\psi}_{i, j+i-1}, & \text { если } j+i-1 \leqslant m, \\
\widetilde{\psi}_{i, j+i-1-m}, & \text { если } j+i-1>m,
\end{array} \quad i, j=1, \ldots, m,\right.
$$

удовлетворяют совокупности сопряженных систем и условиям (0.8), т.е. система для функций $(0.9)$ лежит в классе $\mathbf{W}^{0}(\alpha)$. Функции $(0.11)$ линейно независимы над $\mathbb{C}(z)$ ввиду алгебраической независимости функций $f_{i}(z) \equiv f_{i 1}(z), i=1, \ldots, m$. Для завершения доказательства теоремы I применим теорему II.

Приложения. В качестве иллюстрации применения теоремы II мы воспользуемся классическим результатом Зигеля [4] для значений функции

$$
\begin{aligned}
K_{\lambda}(z) & =\sum_{\nu=0}^{\infty} \frac{(-1)^{\nu}}{\nu !(\lambda+1)_{\nu}}\left(\frac{z}{2}\right)^{2 \nu}, \quad \lambda \in \mathbb{Q} \backslash\{-1,-2, \ldots\}, \\
(\lambda+1)_{0} & =1, \quad(\lambda+1)_{\nu}=(\lambda+1) \cdots(\lambda+\nu), \quad \nu=1,2, \ldots,
\end{aligned}
$$

удовлетворяющей линейному однородному уравнению второго порядка

$$
y^{\prime \prime}+\frac{2 \lambda+1}{z} y^{\prime}+y=0
$$

TEOPEMA III. Пycmb

$$
\begin{aligned}
\lambda_{i} \in \mathbb{Q} \backslash\{-1,-2, \ldots\}, \quad \lambda_{i} \neq \frac{2 \mu-1}{2}, \quad \mu \in \mathbb{Z}, \quad i=1, \ldots, m, \\
\lambda_{i_{1}} \pm \lambda_{i_{2}} \notin \mathbb{Z}, \quad i_{1}, i_{2}=1, \ldots, m, \quad i_{1} \neq i_{2}, \\
\xi_{1}, \ldots, \xi_{n} \in \mathbb{Q} \backslash\{0\}, \quad \xi_{j_{1}}^{2} \neq \xi_{j_{2}}^{2}, \quad j_{1}, j_{2}=1, \ldots, n, \quad j_{1} \neq j_{2} .
\end{aligned}
$$

Тогда существуют положстельные постоянные $C$ и $\gamma$, зависящие только от параметров $\lambda_{1}, \ldots, \lambda_{m}, \xi_{1}, \ldots, \xi_{n}$ и натурального числа $d$, и обладающие следующим свойством: для произвольного (не обязательно однородного) многочлена $P$ от переменных $y_{i j}, i=1, \ldots, m, j=1, \ldots, n$, имеющего иелые коэффициенты и степень $d$, справедливо неравенство

$$
\left.|P|_{y_{i j}=K_{\lambda_{i}}\left(\xi_{j}\right)}|>C| \Pi\right|^{-1} H^{1-\gamma(\log \log H)^{-1 /(m n+2)}}
$$

где П - прочзведение всех ненулевых коэффициентов многочлена $P, H \geqslant 3$ его высcoma. 
ДокАЗАТЕЛЬСТво. Функции $y_{1}=K_{\lambda}(\xi z), y_{2}=K_{\lambda}^{\prime}(\xi z)$ составляют решение системы линейных однородных дифференциальных уравнений

$$
\frac{d}{d z}\left(\begin{array}{l}
y_{1} \\
y_{2}
\end{array}\right)=\left(\begin{array}{cc}
0 & 1 \\
-\xi^{2} & -\frac{2 \lambda+1}{z}
\end{array}\right)\left(\begin{array}{l}
y_{1} \\
y_{2}
\end{array}\right) .
$$

Соответствуюшая сопряженная система имеет вид

$$
\frac{d}{d z}\left(\begin{array}{l}
a_{1} \\
a_{2}
\end{array}\right)=\left(\begin{array}{cc}
0 & \xi^{2} \\
-1 & \frac{2 \lambda+1}{z}
\end{array}\right)\left(\begin{array}{l}
a_{1} \\
a_{2}
\end{array}\right)
$$

Если $a_{1}(z), a_{2}(z)$ - любое решение системы $(0.14)$, то функция $\varphi(z)=a_{1}\left(z / \xi^{2}\right)$ вместе со своей производной $\psi(z)=\varphi^{\prime}(z)=a_{2}\left(z / \xi^{2}\right)$ составляет решение системы

$$
\frac{d}{d z}\left(\begin{array}{c}
\varphi \\
\psi
\end{array}\right)=\left(\begin{array}{cc}
0 & 1 \\
-\frac{1}{\xi^{2}} & \frac{2 \lambda+1}{z}
\end{array}\right)\left(\begin{array}{c}
\varphi \\
\psi
\end{array}\right)
$$

Такую функцию $\varphi(z)$ с условиями $\varphi\left(\xi^{2}\right)=a_{1}(1)=1, \varphi^{\prime}\left(\xi^{2}\right)=a_{2}(1)=0$ обозначим через $\varphi_{\lambda, \xi}(z)$.

Воспользуемся теперь леммой $1[1$, гл. $9, \S 1]:$ при заданном в условии теоремы выборе параметров $\lambda_{1}, \ldots, \lambda_{m}, \xi_{1}, \ldots, \xi_{n}$ функции

$$
\varphi_{\lambda_{i}, \xi_{j}}(z), \quad \varphi_{\lambda_{i}, \xi_{j}}^{\prime}(z), \quad i=1, \ldots, m, \quad j=1, \ldots, n,
$$

алгебраически независимы над $\mathbb{C}(z)$. Но это означает, что система дифференциальных уравнений, которой удовлетворяют функции

$f_{0}(z)=1, f_{i j, 1}(z)=K_{\lambda_{i}}\left(\xi_{j} z\right), f_{i j, 2}(z)=K_{\lambda_{i}}^{\prime}\left(\xi_{j} z\right), \quad i=1, \ldots, m, \quad j=1, \ldots, n$, лежит в классе $\mathbf{W}^{0}(\alpha)$. Для завершения доказательства теоремы III применим теорему II.

ЗАмЕчАниЕ. Условие принадлежности системы $(0.6)$ классу $\mathbf{W}^{0}(\alpha)$ для заданной неособой точки $z=\alpha$ слабее условия нормальности Зигеля для этой системы.

\section{§1. Вспомогательные утверждения}

Ранг числовых линейных форм специального вида. Обозначим через $\mathbb{M}$ модуль линейных форм от переменных $y_{1}, \ldots, y_{m}$ над кольцом $\mathbb{C}[z]$. Таким образом, элементы $R \in \mathbb{M}$ имеют вид

$$
R=\sum_{k=1}^{m} P_{k}(z) y_{k}, \quad P_{k}(z) \in \mathbb{C}[z], \quad k=1, \ldots, m .
$$

Рассмотрим систему линейных однородных дифференциальных уравнений первого порядка

$$
y_{l}^{\prime}=\sum_{j=1}^{m} Q_{l j} y_{j}, \quad Q_{l j}=Q_{l j}(z) \in \mathbb{C}(z), \quad l, j=1, \ldots, m .
$$


Выберем многочлен $T=T(z)$ для системы (1.1) таким образом, что $T Q_{l j} \in \mathbb{C}[z]$, $l, j=1, \ldots, m$.

Определим на $\mathbb{M}$ дифференциальный оператор

$$
D=\frac{\partial}{\partial z}+\sum_{l=1}^{m}\left(\sum_{j=1}^{m} Q_{l j} y_{j}\right) \frac{\partial}{\partial y_{l}}
$$

связанный с системой дифференциальных уравнений (1.1). Если $R \in \mathbb{M}$, то очевидно, что и $T D R \in \mathbb{M}$. Следовательно, $T D: \mathbb{M} \rightarrow \mathbb{M}$. Kроме того $[1$, гл. $3, \S 4]$, если $y_{1}, \ldots, y_{m}$ есть произвольное решение системы (1.1), то

$$
D R=\frac{d}{d z} R=R^{\prime}
$$

Рассмотрим произвольную линейную форму из $\mathbb{M}$

$$
R^{[0]}=\sum_{k=1}^{m} P_{k}^{[0]}(z) y_{k}, \quad P_{k}^{[0]}(z) \in \mathbb{C}[z], \quad k=1, \ldots, m,
$$

и положим

$$
R^{[n+1]}=T D R^{[n]}, \quad n \geqslant 0 .
$$

Пользуясь приведенным выше рассуждением, убеждаемся в том, что $R^{[n]} \in \mathbb{M}$, $n \geqslant 1$, иными словами,

$$
R^{[n]}=\sum_{k=1}^{m} P_{k}^{[n]}(z) y_{k}, \quad P_{k}^{[n]}(z) \in \mathbb{C}[z], \quad n \geqslant 0, \quad k=1, \ldots, m .
$$

С помощью определения (1.2) оператора $D$ и равенства (1.4) находим следующие рекуррентные формулы:

$$
P_{k}^{[n+1]}(z)=T(z)\left(\frac{d}{d z} P_{k}^{[n]}(z)+\sum_{l=1}^{m} P_{l}^{[n]}(z) Q_{l k}(z)\right), \quad n \geqslant 0, \quad k=1, \ldots, m .
$$

Будем пользоваться обозначением $\Omega=\{1, \ldots, m\}$.

В этом пункте мы докажем следующее утверждение.

ПРЕДЛОЖЕНИЕ 1.1. Пусть для заданной линейной формъ (1.3) из $\mathbb{M}$ ранг над $\mathbb{C}(z)$ квадратной матриць

$$
\left(P_{k}^{[n]}(z)\right)_{n=0,1, \ldots, m-1 ; k \in \Omega}
$$

где входящие в нее многочлены определяются согласно формулам (1.5), равен в точности $\widetilde{m}$. Для произвольного подмножества $\widetilde{\Omega} \subset \Omega$, Card $\widetilde{\Omega}=\widetilde{m}$, положим

$$
\Delta(\widetilde{\Omega} ; z)=\operatorname{det}\left(P_{k}^{[n]}(z)\right)_{n=0,1, \ldots, \widetilde{m}-1 ; k \in \widetilde{\Omega}}
$$

Пусть $\alpha \in \mathbb{C}$ - неособая точка системь (1.1), т.е. $T(\alpha) \neq 0$, и известно, что

$$
\max _{\widetilde{\Omega}}\left\{\operatorname{ord}_{z=\alpha} \Delta(\widetilde{\Omega} ; z)\right\}=q
$$

дде максимум берется по всевозможным множествам $\widetilde{\Omega} \subset \Omega, \operatorname{Card} \widetilde{\Omega}=\widetilde{m}$, для которых $\Delta(\widetilde{\Omega} ; z) \not \equiv 0$. Тогда ранг над $\mathbb{C}$ числовой матрицъ

равен в точности $\widetilde{m}$.

$$
\left(P_{k}^{[n]}(\alpha)\right)_{n=0,1, \ldots, \widetilde{m}+q-1 ; k \in \Omega}
$$


ЗАмЕчАнИЕ. На самом деле, в дальнейшем понадобится не сформулированное утверждение, а только механизм его доказательства. Несмотря на это, предложение 1.1 представляется нам содержательным и весьма полезным как обобшение одной леммы Зигеля (см. [1, гл. $3, \S 7$, лемма 10]).

ДокАЗАТЕЛЬСТво. Согласно лемме $7[1$, гл. $3, \S 4]$ фундаментальную систему решений

$$
\left(y_{k \eta}(z)\right)_{k \in \Omega ; \eta=1, \ldots, m}
$$

для (1.1) можно выбрать таким образом, что формы $R^{[0]}, R^{[1]}, R^{[2]}, \ldots$ обрашаются в нуль при подстановке в них вместо переменных $y_{1}, \ldots, y_{m}$ каждого из $m-\widetilde{m}$ решений $y_{1 \eta}, \ldots, y_{m}, \eta=\widetilde{m}+1, \ldots, m$. Отметим сразу, что все функции, входящие в матрицу (1.7), аналитичны в точке $z=\alpha$, поскольку она не является особой точкой системы (1.1).

Для результата подстановки функций $y_{1 \eta}, \ldots, y_{m}, \eta=1, \ldots, m$, вместо переменных $y_{1}, \ldots, y_{m}$ в форму $R^{[n]} \in \mathbb{M}$ введем обозначение

$$
R_{\eta}^{[n]}=R_{\eta}^{[n]}(z)=\sum_{k \in \Omega} P_{k}^{[n]}(z) y_{k \eta}(z), \quad n \geqslant 0, \quad \eta=1, \ldots, \omega .
$$

Тогда согласно выбору матришы (1.7)

$$
R_{\eta}^{[n]}(z) \equiv 0, \quad n \geqslant 0, \quad \eta=\widetilde{m}+1, \ldots, m .
$$

Введем в рассмотрение следующие функции, аналитичные в точке $z=\alpha$ :

$$
\begin{gathered}
\Lambda(z)=\operatorname{det}\left(y_{k \eta}(z)\right)_{k \in \Omega ; \eta=1, \ldots, m}, \\
\lambda(\widetilde{\Omega} ; z)=\operatorname{det}\left(y_{k \eta}(z)\right)_{k \in \Omega \backslash \widetilde{\Omega} ; \eta=\widetilde{m}+1, \ldots, m}, \quad \widetilde{\Omega} \subset \Omega, \quad \operatorname{Card} \widetilde{\Omega}=\widetilde{m}
\end{gathered}
$$

(в случае $\widetilde{m}=m$ считаем $\lambda(\Omega ; z) \equiv 1)$.

Лемма 1.2. Пусть $\mathscr{N}$ - произвольное подмножество $\mathbb{Z}^{+}=\{0,1,2, \ldots\}$ такое, ито $\operatorname{Card} \mathscr{N}=\widetilde{m}$, и $\widetilde{\Omega} \subset \Omega$, Card $\widetilde{\Omega}=\widetilde{m}$. Тогда справедливо тождество

$$
\operatorname{det}\left(P_{k}^{[n]}(z)\right)_{n \in \mathcal{N} ; k \in \widetilde{\Omega}} \cdot \Lambda(z)=\operatorname{det}\left(R_{\eta}^{[n]}(z)\right)_{n \in \mathcal{N} ; \eta=1, \ldots, \widetilde{m}} \cdot \lambda(\widetilde{\Omega} ; z) .
$$

ДоКАЗАТЕЛЬСТво. По правилу умножения матриц и согласно обозначениям (1.8) имеем

$$
\begin{aligned}
& \left(\begin{array}{c}
P_{k}^{[n]}(z) \\
\delta_{i k}
\end{array}\right)_{n \in \mathcal{N}, i \in \Omega \backslash \widetilde{\Omega} ; k \in \Omega}\left(y_{k \eta}(z)\right)_{k \in \Omega ; \eta=1, \ldots, m} \\
& \quad=\left(\begin{array}{c}
R_{\eta}^{[n]}(z) \\
y_{i \eta}(z)
\end{array}\right)_{n \in \mathcal{N}, i \in \Omega \backslash \widetilde{\Omega} ; \eta=1, \ldots, m}
\end{aligned}
$$


где через $\delta_{i k}$ обозначен символ Кронекера. Ввиду равенств (1.9) находим

$$
\begin{aligned}
\operatorname{det} & \left(\begin{array}{c}
R_{\eta}^{[n]}(z) \\
y_{i \eta}(z)
\end{array}\right)_{n \in \mathcal{N}, i \in \Omega \backslash \widetilde{\Omega} ; \eta=1, \ldots, m} \\
= & \operatorname{det}\left(R_{\eta}^{[n]}(z)\right)_{n \in \mathcal{N} ; \eta=1, \ldots, \widetilde{m}} \cdot \operatorname{det}\left(y_{i \eta}(z)\right)_{i \in \Omega \backslash \widetilde{\Omega} ; \eta=\widetilde{m}+1, \ldots, m},
\end{aligned}
$$

а кроме того,

$$
\operatorname{det}\left(\begin{array}{c}
P_{k}^{[n]}(z) \\
\delta_{i k}
\end{array}\right)_{n \in \mathcal{N}, i \in \Omega \backslash \widetilde{\Omega} ; k \in \Omega}=\operatorname{det}\left(P_{k}^{[n]}(z)\right)_{n \in \mathscr{N} ; k \in \widetilde{\Omega}}
$$

Поэтому после перехода от матриц к определителям равенство (1.11) примет вид (1.10).

Лемма 1.3. Если для $\widetilde{\Omega} \subset \Omega, \operatorname{Card} \widetilde{\Omega}=\widetilde{m}$, выполнено $\lambda(\widetilde{\Omega} ; \alpha) \neq 0$, то $\Delta(\widetilde{\Omega} ; z) \not \equiv 0$. Если при этом

$$
\underset{z=\alpha}{\operatorname{ord}} \Delta(\widetilde{\Omega} ; z)=p
$$

то ранг числовой матрищъ

$$
\left(P_{k}^{[n]}(\alpha)\right)_{n=0,1, \ldots, \widetilde{m}+p-1 ; k \in \widetilde{\Omega}}
$$

равен $\widetilde{m}$.

ДокАЗАТЕльство. В случае $\mathscr{N}=\{0,1, \ldots \widetilde{m}-1\}$ согласно тождеству леммы 1.2 имеем

$$
\Delta(\widetilde{\Omega} ; z) \cdot \Lambda(z)=\operatorname{det}\left(R_{\eta}^{[n]}(z)\right)_{n=0,1, \ldots, \widetilde{m}-1 ; \eta=1, \ldots, \widetilde{m}} \cdot \lambda(\widetilde{\Omega} ; z) .
$$

Если предположить, что $\lambda(\widetilde{\Omega} ; z) \not \equiv 0$ и в то же время $\Delta(\widetilde{\Omega} ; z) \equiv 0$, то получим, что $\operatorname{det}\left(R_{\eta}^{[n]}(z)\right)_{n=0,1, \ldots, \widetilde{m}-1 ; \eta=1, \ldots, \widetilde{m}} \equiv 0$. Отсюда и из равенства (1.13) заключаем, что $\Delta(\widetilde{\Omega} ; z) \equiv 0$ для любого множества $\widetilde{\Omega} \subset \Omega$, Card $\widetilde{\Omega}=\widetilde{m}$, иньми словами, что ранг матрицы

$$
\left(P_{k}^{[n]}(z)\right)_{n=0,1, \ldots, \widetilde{m}-1 ; k \in \Omega}
$$

меньше $\widetilde{m}$. С другой стороны, ранг матрицы (1.6) равен $\widetilde{m}$ и по лемме $6[1$, гл.3, $\S 4]$ ранг матрицы (1.14) также равен $\widetilde{m}$. Полученное противоречие означает, что в случае $\lambda(\widetilde{\Omega} ; z) \not \equiv 0$ имеем $\Delta(\widetilde{\Omega} ; z) \not \equiv 0$.

Перейдем к доказательству второй части леммы. Для заданного множества $\widetilde{\Omega}$ положим $\Delta(z)=\Delta(\widetilde{\Omega} ; z)$ и перепишем равенство $(1.13)$ в виде

$$
\Delta(z) \cdot \chi(z)=\operatorname{det}\left(R_{\eta}^{[n]}(z)\right)_{n=0,1, \ldots, \widetilde{m}-1 ; \eta=1, \ldots, \widetilde{m}},
$$


где $\chi(z)=\Lambda(z) / \lambda(\widetilde{\Omega} ; z)$ - аналитическая в точке $z=\alpha$ функция, поскольку $\lambda(\widetilde{\Omega} ; \alpha) \neq 0$. Перепишем формулы (1.4) для функций (1.8):

$$
R_{\eta}^{[n+1]}(z)=T(z) \frac{d}{d z} R_{\eta}^{[n]}(z), \quad n \geqslant 0, \quad \eta=1, \ldots, \widetilde{m}
$$

Пользуясь соотношениями (1.15), (1.16), правилом дифференцирования определителя по строкам и тождеством леммы 1.2 , получаем

$$
\begin{gathered}
\left(T(z) \frac{d}{d z}\right)^{p}(\Delta(z) \chi(z))=\left(T(z) \frac{d}{d z}\right)^{p} \operatorname{det}\left(R_{\eta}^{[n]}(z)\right)_{n=0,1, \ldots, \widetilde{m}-1 ; \eta=1, \ldots, \widetilde{m}} \\
=\sum_{\substack{\nu_{0}+\cdots+\nu_{\widetilde{m}-1}=p \\
\nu_{0}, \ldots, \nu_{\widetilde{m}-1} \geqslant 0}} \frac{p !}{\nu_{0} ! \cdots \nu_{\widetilde{m}-1} !} \operatorname{det}\left(R_{\eta}^{\left[n+\nu_{n}\right]}(z)\right)_{n=0,1, \ldots, \widetilde{m}-1 ; \eta=1, \ldots, \widetilde{m}} \\
=\sum_{\substack{\nu_{0}+\cdots+\nu_{\widetilde{m}-1}=p \\
\nu_{0}, \ldots, \nu_{\widetilde{m}-1} \geqslant 0}} \frac{p !}{\nu_{0} ! \cdots \nu_{\widetilde{m}-1} !} \operatorname{det}\left(P_{k}^{\left[n+\nu_{n}\right]}(z)\right)_{n=0,1, \ldots, \widetilde{m}-1 ; k \in \widetilde{\Omega}} \cdot \chi(z) . \\
\end{gathered}
$$

Отметим, что последнее тождество в случае $\widetilde{m}=m$ было доказано в дипломной работе В.В. Титенко в 1987 году. Функция $\chi(z)$ в точке $z=\alpha$ аналитична и принимает ненулевое значение; по условию $T(\alpha) \neq 0$. Поэтому, если $p$-точньй порядок нуля в точке $z=\alpha$ многочлена $\Delta(z)$, то подстановка $z=\alpha$ в тождество (1.17) с последуюшим делением обеих частей на $\chi(\alpha)$ даст соотношение

$$
0 \neq T^{p}(\alpha) \Delta^{(p)}(\alpha)=\sum_{\substack{\nu_{0}+\cdots+\nu_{\widetilde{m}-1}=p \\ \nu_{0}, \ldots, \nu_{\tilde{m}-1} \geqslant 0}} \frac{p !}{\nu_{0} ! \cdots \nu_{\widetilde{m}-1} !} \operatorname{det}\left(P_{k}^{\left[n+\nu_{n}\right]}(\alpha)\right)_{n=0,1, \ldots, \widetilde{m}-1 ; k \in \widetilde{\Omega}}
$$

Отсюда следует, что для некоторого набора $\nu_{0}, \ldots, \nu_{\widetilde{m}-1}, \nu_{0}+\cdots+\nu_{\widetilde{m}-1}=p$, неотрицательных целых чисел вьполнено

$$
\operatorname{det}\left(P_{k}^{\left[n+\nu_{n}\right]}(\alpha)\right)_{n=0,1, \ldots, \widetilde{m}-1 ; k \in \widetilde{\Omega}} \neq 0
$$

а это, в свою очередь, означает, что ранг числовой матрицы (1.12) в точности равен $\widetilde{m}$.

Вернемся теперь к доказательству предложения 1.1.

Если предположить, что для любого множества $\widetilde{\Omega} \subset \Omega, \operatorname{Card} \widetilde{\Omega}=\widetilde{m}$, выполнено $\lambda(\widetilde{\Omega} ; \alpha)=0$, то согласно определению миноров $\lambda(\widetilde{\Omega} ; z)$ столбцы числовой матрицы $\left(y_{k \eta}(\alpha)\right)_{k \in \widetilde{\Omega} ; \eta=\widetilde{m}+1, \ldots, m}$ линейно зависимы. Это, однако, противоречит тому, что матрица (1.7) является фундаментальной матрищей решений системы (1.1), для которой точка $z=\alpha$ является неособой. Поэтому $\lambda(\widetilde{\Omega} ; \alpha) \neq 0$, по крайней мере, для одного такого $\widetilde{\Omega}$. Остается воспользоваться леммой 1.3 и тем фактом, что $p \leqslant q$. 
Лемма Галочкина. Излагаемое ниже утверждение является одним из ключевых моментов описываемого в работе метода. Его любезно предоставил автору А.И. Галочкин.

ЛЕмма 1.4 (А.И. Галочкин). Пусть

$$
\begin{gathered}
\Delta(z)=\sum_{\nu=p}^{s} \frac{\Delta_{\nu}}{\nu !} z^{\nu}, \quad \Delta_{\nu} \in \mathbb{Z}, \quad\left|\Delta_{\nu}\right| \leqslant \delta, \quad \nu=p, p+1, \ldots, s \\
s=\operatorname{deg} \Delta(z), \quad p=\operatorname{ord}_{z=0} \Delta(z) \geqslant 2, \quad q=\operatorname{ord}_{z=\alpha} \Delta(z), \quad \alpha \in \mathbb{C}, \quad|\alpha| \leqslant \frac{p-\sqrt{p}}{1+\sqrt{p}} .
\end{gathered}
$$

Тогда

$$
q \leqslant \frac{2 \log (s \delta)}{\log p}
$$

ДокАЗАТЕЛЬСТво. При $|\xi|=p$ имеем

$$
|\Delta(\xi)| \leqslant(s-p+1) \frac{\delta}{p !} p^{p},
$$

где использовано неравенство

$$
\frac{p^{\nu}}{\nu !} \leqslant \frac{p^{p}}{p !} \quad \text { при } \quad \nu \geqslant p .
$$

Поскольку

$$
\Delta(z)=\frac{1}{2 \pi i} \oint_{|\xi|=p} \frac{z^{p}(z-\alpha)^{q}}{\xi^{p}(\xi-\alpha)^{q}} \frac{\Delta(\xi)}{\xi-z} d \xi
$$

а также

$$
\frac{s-p+1}{p-1} \leqslant \frac{s}{p}
$$

при $|z|=1$ получаем

$$
\begin{aligned}
|\Delta(z)| & \leqslant \frac{1}{2 \pi} \oint_{|\xi|=p} \frac{(1+|\alpha|)^{q}}{p^{p}(p-|\alpha|)^{q}} \frac{s-p+1}{p-1} \frac{\delta}{p !} p^{p} d \xi \leqslant s \delta\left(\frac{1+|\alpha|}{p-|\alpha|}\right)^{q} \frac{1}{p !} \\
& \leqslant s \delta\left(\frac{1}{\sqrt{p}}\right)^{q} \frac{1}{p !} .
\end{aligned}
$$

Далее имеем

$$
\Delta_{p}=\frac{p !}{2 \pi i} \oint_{|z|=1} \frac{\Delta(z)}{z^{p+1}} d z
$$

откуда

$$
\left|\Delta_{p}\right| \leqslant p ! \cdot \max _{|z|=1}|\Delta(z)| \leqslant s \delta p^{-q / 2} .
$$

С другой стороны, число $\Delta_{p}$ является целым и отлично от нуля, т.е. $\left|\Delta_{p}\right| \geqslant 1$, откуда

$$
p^{q / 2} \leqslant s \delta
$$


что после логарифмирования дает неравенство (1.18). Лемма доказана.

Представление функции $g(z)$ в виде степенного ряда

$$
g(z)=\sum_{\nu=0}^{\infty} \frac{g_{\nu}}{\nu !} z^{\nu}, \quad g_{\nu} \in \mathbb{C},
$$

назовем нормальным разложением функиии, а числа $g_{\nu}, \nu \in \mathbb{Z}^{+},-$коэффиииентами в нормальном разложсении функиии. Если при этом функция $g(z)$ является многочленом, т.е. степенной ряд (1.19) содержит лиш конечное количество слагаемых, то будем полагать

$$
\|g(z)\|=\max _{\nu}\left\{\left|g_{\nu}\right|\right\} .
$$

ЛЕмма 1.5 [8]. Для многочлена $g(z)$ справедливы следующие неравенства:

а) $\left\|g^{\prime}(z)\right\| \leqslant\|g(z)\|$;

б) $\left\|g_{1}(z)+g_{2}(z)\right\| \leqslant\left\|g_{1}(z)\right\|+\left\|g_{2}(z)\right\|$;

в) $\left\|g_{1}(z) g_{2}(z)\right\| \leqslant\left(\begin{array}{c}\operatorname{deg} g_{1}(z)+\operatorname{deg} g_{2}(z) \\ \operatorname{deg} g_{1}(z)\end{array}\right)\left\|g_{1}(z)\right\| \cdot\left\|g_{2}(z)\right\|$.

Приведем теперь необходимое в дальнейшем следствие из леммы 1.4.

ЛЕМма 1.6. Пусть для функции

$$
\Delta(z)=\operatorname{det}\left(P_{n k}(z)\right)_{n, k=1, \ldots, \widetilde{m}},
$$

где многочлень $P_{n k}(z) \in \mathbb{C}[z]$ имеют челье коэффициенты в нормальном разложении и удовлетворяют условиям

$$
\operatorname{deg} P_{n k}(z) \leqslant d, \quad\left\|P_{n k}(z)\right\| \leqslant H, \quad n, k=1, \ldots, \widetilde{m},
$$

известно, что

$$
\operatorname{ord}_{z=0} \Delta(z) \geqslant p
$$

число р достаточно велико, точка $z=\alpha$ фиксирована. Тогда

$$
\underset{z=\alpha}{\operatorname{ord}} \Delta(z)<\frac{2 \widetilde{m}}{\log p}(\log H+2 d(1+\log \tilde{m})) .
$$

ДокАЗАтЕльство. Раскрывая $\Delta(z)$ как определитель, по лемме 1.5 получим

$$
\begin{aligned}
\|\Delta(z)\| & \leqslant \sum_{\sigma \in \mathbf{S}_{\widetilde{m}}}\left\|P_{1 \sigma(1)}(z) P_{2 \sigma(2)}(z) \cdots P_{\widetilde{m} \sigma(\widetilde{m})}(z)\right\| \leqslant \widetilde{m} ! \cdot \frac{(\widetilde{m} d) !}{(d !)^{\widetilde{m}}} \cdot H^{\widetilde{m}} \\
& \leqslant \widetilde{m}^{\widetilde{m}} \cdot \frac{(\widetilde{m} d)^{\widetilde{m} d}}{(d / e)^{\widetilde{m} d}} \cdot H^{\widetilde{m}}=\widetilde{m}^{\widetilde{m}(d+1)} e^{\widetilde{m} d} H^{\widetilde{m}} .
\end{aligned}
$$

Осталось воспользоваться леммой 1.4 и тем фактом, что $\operatorname{deg} \Delta(z) \leqslant \widetilde{m} d<e^{\widetilde{m} d}$ :

$$
\begin{aligned}
\underset{z=\alpha}{\operatorname{ord}} \Delta(z) & \leqslant \frac{2 \log (\operatorname{deg} \Delta(z) \cdot\|\Delta(z)\|)}{\log p}<\frac{2 \log \left(\widetilde{m}^{\widetilde{m}(d+1)} e^{2 \widetilde{m} d} H^{\widetilde{m}}\right)}{\log p} \\
& \leqslant \frac{2 \widetilde{m}}{\log p}(\log H+2 d(1+\log \widetilde{m}))
\end{aligned}
$$

что и требовалось. 


\section{§2. Градуированные приближения Паде}

Для доказательства теоремы II воспользуемся упомянутой выше конструкцией работы [7].

Обозначим через $T(z)$ наименшший общий знаменатель рациональных коэффициентов системы $(0.5)$ :

$$
T(z) \in \mathbb{Z}[z], \quad T(z) Q_{l j}^{(i)}(z) \in \mathbb{Z}[z], \quad l, j=1, \ldots, m_{i}, \quad i=1, \ldots, m
$$

Системы приближающих функциональных форм, строящиеся ниже, будут зависеть от натуральных параметров $M_{\bar{u}}, \bar{u} \in U$. Положим

$$
\begin{gathered}
M=\max _{\bar{u} \in U}\left\{M_{\bar{u}}\right\}, \quad N=\left[(\log M)^{1 /\left(m_{1}+m_{2}+\cdots+m_{m}-m+2\right)}\right]>d, \\
\varepsilon=\sum_{\bar{u} \in U} \sum_{i=1}^{m} \sum_{v=1}^{u_{i}} \frac{m_{i}-1}{N+m_{i}-v} \asymp \frac{1}{N}, \quad \min _{\bar{u} \in U}\left\{M_{\bar{u}}\right\} \geqslant 3 \varepsilon M,
\end{gathered}
$$

при этом будем считать $M$ достаточно большим. (Здесь и далее квадратньми скобками мы обозначаем целую часть числа.) Буквы $C$ с нижними индексами и $M$ со штрихами будем использовать для обозначения положительных постоянных, зависящих только от функций (0.9), системы (0.5), чисел $\alpha$ и $d$. Положим также $\bar{a}=\left(\bar{a}_{1}, \ldots, \bar{a}_{m}\right)$, где $\bar{a}_{i}=\left(a_{i 1}, \ldots, a_{i m_{i}}\right), i=1, \ldots, m$, и $\bar{\kappa}=\left(\bar{\kappa}_{1}, \ldots, \bar{\kappa}_{m}\right)-$ мультииндекс, где $\bar{\kappa}_{i}=\left(\kappa_{i 1}, \ldots, \kappa_{i m_{i}}\right), i=1, \ldots, m$, причем все компоненты $\kappa_{i j}$ мультииндекса неотрицательны, а если в некоторой сумме встретилось слагаемое хотя бы с одной компонентой $\kappa_{i j}<0$, то считаем это слагаемое отсутствующим (равным нулю). Для экономии места в формулах будем писать

$$
\bar{a}^{\bar{\kappa}}=\prod_{\substack{i=1, \ldots, m \\ j=1, \ldots, m_{i}}} a_{i j}^{\kappa_{i j}}, \quad\left|\bar{\kappa}_{i}\right|=\sum_{j=1}^{m_{i}} \kappa_{i j}, \quad i=1, \ldots, m .
$$

Введем множества

$$
\begin{gathered}
\Omega_{\bar{u}}=\Omega_{\bar{u}}(N)=\left\{\bar{\kappa}:\left|\bar{\kappa}_{i}\right|=N-u_{i}, i=1, \ldots, m\right\}, \quad \bar{u} \in U, \\
\Omega=\Omega(N)=\bigcup_{\bar{u} \in U} \Omega_{\bar{u}}, \quad \Theta=\Theta(N)=\left\{\bar{s}:\left|\bar{s}_{i}\right|=N, i=1, \ldots, m\right\} ;
\end{gathered}
$$

соответствующими маленькими буквами обозначим количества элементов в них $[1$, гл. $2, \S 7$, лемма 7$]$ :

$$
\begin{gathered}
\omega_{\bar{u}}=\operatorname{Card} \Omega_{\bar{u}}=\prod_{i=1}^{m}\left(\begin{array}{c}
N+m_{i}-1-u_{i} \\
m_{i}-1
\end{array}\right), \quad \bar{u} \in U, \\
\omega=\operatorname{Card} \Omega=\sum_{\bar{u} \in U} \omega_{\bar{u}}, \quad \theta=\operatorname{Card} \Theta=\prod_{i=1}^{m}\left(\begin{array}{c}
N+m_{i}-1 \\
m_{i}-1
\end{array}\right) .
\end{gathered}
$$


Искомые линейные формы имеют вид

$$
R(z ; \bar{a})=\sum_{\bar{u} \in U} P_{\bar{u}}(z ; \bar{a}) \prod_{i=1}^{m}\left(a_{i 1} f_{i 1}(z)+\cdots+a_{i m_{i}} f_{i m_{i}}(z)\right)^{N-u_{i}}
$$

где многочлены $P_{\bar{u}}(z ; \bar{a})$ однородны по каждой компоненте $\bar{a}_{i}, i=1, \ldots, m$, и имеют вид

$$
P_{\bar{u}}(z ; \bar{a})=\sum_{\bar{\kappa} \in \Omega_{\bar{u}}} \bar{a}^{\bar{\kappa}} P_{\bar{\kappa}}(z), \quad \bar{u} \in U
$$

Функциональную линейную форму (2.3) можно представить в виде

$$
R(z ; \bar{a})=\sum_{\bar{s} \in \Theta} \bar{a}^{\bar{s}} R_{\bar{s}}(z),
$$

где

$$
R_{\bar{s}}(z)=\sum_{\bar{u} \in U} \sum_{\substack{\bar{r}=\left(\bar{r}_{1}, \ldots, \bar{r}_{m}\right) \\\left|\bar{r}_{i}\right|=u_{i}, i=1, \ldots, m}} \prod_{i=1}^{m} \frac{u_{i} !}{r_{i 1} ! \cdots r_{i m_{i}} !} P_{\bar{s}-\bar{r}}(z) \bar{f}^{\bar{r}}(z), \quad \bar{s} \in \Theta .
$$

Лемма 2.1. Для натуральных $M_{\bar{u}}, \bar{u} \in U$, и выбранных согласно (2.2) чисел $M, N$ и с существуют многочленьи $P_{\bar{\kappa}}(z) \in \mathbb{Q}[z], \bar{\kappa} \in \Omega$, удовлетворяющие следующим условиям:

1) не все они тохсдественно равны нулю;

2) $\operatorname{deg} P_{\bar{\kappa}}<M$ для всех $\bar{\kappa} \in \Omega$;

3) $\underset{z=0}{\operatorname{ord}} P_{\bar{\kappa}} \geqslant M-M_{\bar{u}}$ для всех $\bar{\kappa} \in \Omega_{\bar{u}}, \bar{u} \in U$;

4) коэффициенты в нормальном разложении этих многочленов являются иелыми числами, ограниченными по модулю величиной $C_{0}^{M / \varepsilon}$;

5) порядок нуля в точке $z=0$ каждой из линейных функииональньх форм (2.6) не ниже

$$
K=\left[\sum_{\bar{u} \in U} \frac{\omega_{\bar{u}}}{\theta} M_{\bar{u}}-\varepsilon M\right] .
$$

ДоКАЗАТЕЛЬСТво этой леммы проводится по той же схеме, что и доказательство леммы 1.1 [8].

ЗАМЕчанИЕ 1. Имеем

$$
\begin{aligned}
\frac{\omega_{\bar{u}}}{\theta} & =\prod_{i=1}^{m} \frac{\left(\begin{array}{c}
N+m_{i}-1-u_{i} \\
m_{i}-1
\end{array}\right)}{\left(\begin{array}{c}
N+m_{i}-1 \\
m_{i}-1
\end{array}\right)}=\prod_{i=1}^{m} \frac{\left(N+m_{i}-1-u_{i}\right) !}{\left(N+m_{i}-1\right) !} \cdot \frac{N !}{\left(N-u_{i}\right) !} \\
& =\prod_{i=1}^{m} \frac{\left(N-u_{i}+1\right) \cdots(N-1) N}{\left(N+m_{i}-u_{i}\right) \cdots\left(N+m_{i}-2\right)\left(N+m_{i}-1\right)} \quad \bar{u} \in U, \\
& =\prod_{i=1}^{m} \prod_{v=1}^{u_{i}}\left(1-\frac{m_{i}-1}{N+m_{i}-v}\right)>1-\sum_{i=1}^{m} \sum_{v=1}^{u_{i}} \frac{m_{i}-1}{N+m_{i}-v}, \quad \bar{u}
\end{aligned}
$$


откуда

$$
\begin{aligned}
K & =\left[\sum_{\bar{u} \in U} \frac{\omega_{\bar{u}}}{\theta} M_{\bar{u}}-\varepsilon M\right]=\left[\sum_{\bar{u} \in U} M_{\bar{u}}-\sum_{\bar{u} \in U}\left(1-\frac{\omega_{\bar{u}}}{\theta}\right) M_{\bar{u}}-\varepsilon M\right] \\
& >\left[\sum_{\bar{u} \in U} M_{\bar{u}}-\sum_{\bar{u} \in U} \sum_{i=1}^{m} \sum_{v=1}^{u_{i}} \frac{m_{i}-1}{N+m_{i}-v} M-\varepsilon M\right]=\left[\sum_{\bar{u} \in U} M_{\bar{u}}-2 \varepsilon M\right] .
\end{aligned}
$$

Последнее неравенство дает “более наглядное" представление величины $K$.

ЗАмЕчАнИЕ 2. В качестве дополнения к лемме 2.1 требует обоснования факт $R(z ; \bar{a}) \not \equiv 0$ для построенной формы $R(z ; \bar{a})$. Среди всех мультииндексов $\bar{s} \in \Theta$ таких, что в наборе

$$
P_{\bar{s}-\bar{r}}(z), \quad\left|\bar{r}_{i}\right|=u_{i}, \quad i=1, \ldots, m
$$

есть хотя бы один ненулевой многочлен, выберем $\bar{s}^{\prime}$ с наибольшей возможной суммой $s_{11}^{\prime}+s_{21}^{\prime}+\cdots+s_{m 1}^{\prime}$. Тогда

$$
R_{\bar{s}^{\prime}}(z)=\sum_{\bar{u} \in U} P_{\bar{s}^{\prime}-u_{1} \bar{e}_{11}-u_{2} \bar{e}_{21}-\cdots-u_{m} \bar{e}_{m 1}}(z) f_{11}^{u_{1}}(z) f_{21}^{u_{2}}(z) \cdots f_{m 1}^{u_{m}}(z) \not \equiv 0
$$

ввиду линейной независимости функций $(0.11)$ над $\mathbb{C}(z)$ и согласно выбору $\bar{s}^{\prime} \in \Theta$. Отсюда и вытекает, что $R(z ; \bar{a}) \not \equiv 0$. (Через $\bar{e}_{i j}$ мы обозначаем мультииндекс, у которого на месте с номером $i j$ стоит единица, а на остальных местах - нули.)

Для размножения построенной согласно лемме 2.1 формы $R(z ; \bar{a})$ в формы того же вида введем линейный диффференциальный оператор

$$
D=\frac{\partial}{\partial z}-\sum_{i=1}^{m}\left(\sum_{j=1}^{m_{i}}\left(\sum_{l=1}^{m_{i}} Q_{l j}^{(i)}(z) a_{i l}\right) \frac{\partial}{\partial a_{i j}}\right)
$$

связанный с совокупностью систем линейных однородных дифференциальных уравнений (0.6). Тогда

$$
\begin{aligned}
D \sum_{j=1}^{m_{i}} a_{i j} f_{i j}(z) & =\sum_{j=1}^{m_{i}} a_{i j} \frac{\partial f_{i j}}{\partial z}(z)-\sum_{j=1}^{m_{i}}\left(\sum_{l=1}^{m_{i}} Q_{l j}^{(i)}(z) a_{i l}\right) f_{i j}(z) \\
& =\sum_{l=1}^{m_{i}} a_{i l} \frac{\partial f_{i l}}{\partial z}(z)-\sum_{l=1}^{m_{i}} a_{i l} \sum_{j=1}^{m_{i}} Q_{l j}^{(i)}(z) f_{i j}(z) \\
& =\sum_{l=1}^{m_{i}} a_{i l}\left(f_{i l}^{\prime}(z)-\sum_{j=1}^{m_{i}} Q_{l j}^{(i)}(z) f_{i j}(z)\right)=0, \quad i=1, \ldots, m .
\end{aligned}
$$

Поэтому функциональные формы вида (2.3) после применения к ним оператора $D$ и умножения на $T(z)$ переходят в формы того же вида (с другими коэффициентами-многочленами $\left.P_{\bar{\kappa}}(z), \bar{\kappa} \in \Omega\right)$. 
Положим

$$
\begin{array}{ll}
P_{\bar{\kappa}}^{[0]}(z)=P_{\bar{\kappa}}(z), \quad \bar{\kappa} \in \Omega, \\
R_{\bar{s}}^{[0]}(z)=R_{\bar{s}}(z), \quad \bar{s} \in \Theta,
\end{array}
$$

где $P_{\bar{\kappa}}(z), \bar{\kappa} \in \Omega$, и $R_{\bar{s}}(z), \bar{s} \in \Theta,-$ построенные в лемме 2.1 многочлены и отвечающие им функции $(2.6)$,

$$
R^{[0]}(z ; \bar{a})=\sum_{\bar{s} \in \Theta} \bar{a}^{\bar{s}} R_{\bar{s}}^{[0]}(z) .
$$

Тогда функциональные формы

$$
R^{[n]}(z ; \bar{a})=(T(z) D)^{n} R^{[0]}(z ; \bar{a}), \quad n \geqslant 0,
$$

имеют вид

$$
R^{[n]}(z ; \bar{a})=\sum_{\bar{u} \in U} P_{\bar{u}}^{[n]}(z ; \bar{a}) \prod_{i=1}^{m}\left(a_{i 1} f_{i 1}(z)+\cdots+a_{i m_{i}} f_{i m_{i}}(z)\right)^{N-u_{i}}, \quad n \geqslant 0,
$$

и входящие в них многочлены от $z$ удовлетворяют следующим рекуррентным соотношениям:

$$
\begin{gathered}
P_{\bar{\kappa}}^{[n+1]}(z)=T(z)\left(\frac{d}{d z} P_{\bar{\kappa}}^{[n]}(z)-\sum_{i=1}^{m} \sum_{l, j=1}^{m_{i}}\left(\kappa_{i j}-\delta_{l j}+1\right) Q_{l j}^{(i)}(z) P_{\bar{\kappa}-\bar{e}_{i l}+\bar{e}_{i j}}^{[n]}(z)\right), \\
n \geqslant 0, \quad \bar{\kappa} \in \Omega .
\end{gathered}
$$

Аналогичным соотношениям удовлетворяют и отвечаюшие каждой функциональной форме

$$
R^{[n]}(z ; \bar{a})=\sum_{\bar{s} \in \Theta} \bar{a}^{\bar{s}} R_{\bar{s}}^{[n]}(z), \quad n \geqslant 0,
$$

многочлены $R_{\bar{s}}^{[n]}(z), \bar{s} \in \Theta, n \geqslant 0$, от функций $(0.9)$ :

$$
\begin{gathered}
R_{\bar{s}}^{[n+1]}(z)=T(z)\left(\frac{d}{d z} R_{\bar{s}}^{[n]}(z)-\sum_{i=1}^{m} \sum_{l, j=1}^{m_{i}}\left(s_{i j}-\delta_{l j}+1\right) Q_{l j}^{(i)}(z) R_{\bar{s}_{-}-\bar{e}_{i l}+\bar{e}_{i j}}^{[n]}(z)\right), \\
n \geqslant 0, \quad \bar{s} \in \Theta .
\end{gathered}
$$

Если ввести обозначение

$$
t=\max \left\{\operatorname{deg} T, \max _{i, l, j}\left\{\operatorname{deg} T Q_{l j}^{(i)}\right\}\right\},
$$

то из леммы 2.1 и соотношений $(2.8),(2.9)$ имеем:

$$
\begin{array}{ll}
\operatorname{deg} P_{\bar{\kappa}}^{[n]}<M+t n, & n \geqslant 0, \quad \bar{\kappa} \in \Omega, \\
\operatorname{ord}_{z=0} P_{\bar{\kappa}}^{[n]} \geqslant M-M_{\bar{u}}-n, & n \geqslant 0, \quad \bar{\kappa} \in \Omega_{\bar{u}}, \quad \bar{u} \in U, \\
\operatorname{ord}_{z=0} R_{\bar{s}}^{[n]} \geqslant K-n, & n \geqslant 0, \quad \bar{s} \in \Theta .
\end{array}
$$


ЛЕмма 2.2. а) Коэффициенть в нормальном разложсении многочленов $P_{\bar{\kappa}}^{[n]}(z), n \geqslant 0, \bar{\kappa} \in \Omega$, являются чельми числами. Кроме того, справедливь оценки

$$
\max _{\bar{\kappa} \in \Omega}\left\{\left\|P_{\bar{\kappa}}^{[n]}(z)\right\|\right\} \leqslant\left(C_{1} N\right)^{n} \frac{(M+t n-1) !}{(M-1) !} \cdot C_{0}^{M / \varepsilon}, \quad n \geqslant 0
$$

откуда при $n<C_{2} \varepsilon M$

$$
\max _{\bar{\kappa} \in \Omega}\left\{\left\|P_{\bar{\kappa}}^{[n]}(z)\right\|\right\}<M^{C_{3} \varepsilon M}
$$

б) При $n<C_{2} \varepsilon M$ вьиолняются неравенства

$$
\left|R_{\bar{s}^{*}}^{[n]}(\alpha)\right|<M^{C_{4} \varepsilon M-K}, \quad \bar{s}^{*}=N\left(\bar{e}_{11}+\bar{e}_{21}+\cdots+\bar{e}_{m 1}\right) .
$$

ДокАЗАтЕльство. а) Первая часть утверждения вытекает из соотношений (2.8) и выбора (2.1) многочлена $T(z)$. Положим

$$
C_{5}=\left(1+\sum_{i=1}^{m} m_{i}^{2}\right) \cdot \max \left\{\|T\|, \max _{i, l, j}\left\{\left\|T Q_{l j}^{(i)}\right\|\right\}\right\}
$$

и воспользуемся рекуррентными соотношениями (2.8) и неравенствами леммы 1.5:

$$
\max _{\bar{\kappa} \in \Omega}\left\{\left\|P_{\bar{\kappa}}^{[n+1]}(z)\right\|\right\} \leqslant\left(\begin{array}{c}
M+t(n+1)-1 \\
t
\end{array}\right) \cdot C_{5} N \cdot \max _{\bar{\kappa} \in \Omega}\left\{\left\|P_{\bar{\kappa}}^{[n]}(z)\right\|\right\}, \quad n \geqslant 0,
$$

откуда простая индукция по $n$ дает оценку

$$
\begin{aligned}
\max _{\bar{\kappa} \in \Omega}\left\{\left\|P_{\bar{\kappa}}^{[n]}(z)\right\|\right\} & \leqslant\left(\frac{C_{5} N}{t !}\right)^{n} \frac{(M+t n-1) !}{(M-1) !} \cdot \max _{\bar{\kappa} \in \Omega}\left\{\left\|P_{\bar{\kappa}}^{[0]}(z)\right\|\right\} \\
& \leqslant\left(\frac{C_{5} N}{t !}\right)^{n} \frac{(M+t n-1) !}{(M-1) !} \cdot C_{0}^{M / \varepsilon}, \quad n \geqslant 0
\end{aligned}
$$

а значит, справедливы оценки (2.13). Неравенства (2.14) получаются из (2.13) с учетом

$$
\frac{(M+t n-1) !}{(M-1) !} \cdot N^{n}<(2 M)^{t n} \cdot M^{n}, \quad C_{0}^{M / \varepsilon}=o\left(M^{\varepsilon M}\right) \quad \text { при } \quad M \rightarrow \infty
$$

и $n<C_{2} \varepsilon M$.

б) Пусть $P_{\bar{\kappa}, \nu}^{[n]}, \nu \in \mathbb{Z}^{+},-$коэффициенты в нормальном разложении многочленов $P_{\bar{\kappa}}^{[n]}(z), n \geqslant 0, \bar{\kappa} \in \Omega$, соответственно; $R_{\bar{s}^{*}, \mu}^{[n]}, \mu \in \mathbb{Z}^{+},-$коэффицциенты в нормальном разложении форм $R_{\bar{s}^{*}}^{[n]}(z), n \geqslant 0 ; F_{\bar{u}, \nu}, \nu \in \mathbb{Z}^{+}, \bar{u} \in U,-$ коэффициенты в нормальном разложении функций (0.11). Тогда согласно формуле (2.6)

$$
R_{\bar{s}^{*}}^{[n]}(z)=\sum_{\bar{u} \in U} P_{\bar{s}^{*}-u_{1} \bar{e}_{11}-u_{2} \bar{e}_{21}-\cdots-u_{m} \bar{e}_{m 1}}(z) F_{\bar{u}}(z), \quad n \geqslant 0
$$


и значит,

$$
R_{\bar{s}^{*}, \mu}^{[n]}=\sum_{\bar{u} \in U} \sum_{\nu=0}^{\mu}\left(\begin{array}{l}
\mu \\
\nu
\end{array}\right) P_{\bar{s}^{*}-u_{1} \bar{e}_{11}-u_{2} \bar{e}_{21}-\cdots-u_{m} \bar{e}_{m 1}, \nu}^{[n]} F_{\bar{u}, \mu-\nu}, \quad \mu \in \mathbb{Z}^{+}, \quad n \geqslant 0 .
$$

При этом

$$
R_{\bar{s}^{*}, \mu}^{[n]}=0, \quad \mu<K-n, \quad n<C_{2} \varepsilon M
$$

Поэтому, если в соотношении (2.16) воспользоваться оценками (2.14) и определением $E$-функции для совокупности $(0.11)$, то получаем (при $\mu \geqslant K-n)$

$$
\left|R_{\bar{s}^{*}, \mu}^{[n]}\right| \leqslant \operatorname{Card} U \cdot \sum_{\nu=0}^{\mu}\left(\begin{array}{l}
\mu \\
\nu
\end{array}\right) \max _{\bar{\kappa} \in \Omega}\left\{\left\|P_{\bar{\kappa}}^{[n]}(z)\right\|\right\} C^{\mu+1}<C_{6}^{\mu+1} M^{C_{3} \varepsilon M}
$$

откуда

$$
\left|R_{\bar{s}^{*}}^{[n]}(\alpha)\right|=\left|\sum_{\mu \geqslant K-n} \frac{R_{\bar{s}^{*}, \mu}^{[n]}}{\mu !} \alpha^{\mu}\right|<M^{C_{3} \varepsilon M} \sum_{\mu \geqslant K-n} \frac{|\alpha|^{\mu}}{\mu !} C_{6}^{\mu+1} .
$$

Пользуясь неравенством

$$
\begin{aligned}
\sum_{\mu \geqslant K-n} \frac{|\alpha|^{\mu}}{\mu !} C_{6}^{\mu+1} & \leqslant \frac{|\alpha|^{K-n} C_{6}^{K-n+1}}{(K-n) !} \sum_{\mu \geqslant K-n} \frac{\left(C_{6}|\alpha|\right)^{\mu-K+n}}{(\mu-K+n) !} \\
& =\frac{|\alpha|^{K-n} C_{6}^{K-n+1}}{(K-n) !} e^{C_{6}|\alpha|} \\
& <\left(C_{6}|\alpha|\right)^{\operatorname{Card} U \cdot M}\left(\frac{e}{K-n}\right)^{K-n} e^{C_{6}|\alpha|} \\
& <\left(C_{6}|\alpha|\right)^{\operatorname{Card} U \cdot M}\left(\frac{e}{M}\right)^{K} e^{C_{6}|\alpha|} \\
& <e^{C_{6}|\alpha|}\left(C_{6}|\alpha| e\right)^{\operatorname{Card} U \cdot M} M^{-K}
\end{aligned}
$$

поскольку $M<K-n \leqslant K<\operatorname{Card} U \cdot M$, получаем

$$
\left|R_{\bar{s}^{*}}^{[n]}(\alpha)\right|<M^{C_{3} \varepsilon M} e^{C_{6}|\alpha|}\left(C_{6}|\alpha| e\right)^{\operatorname{Card} U \cdot M} M^{-K}
$$

Последняя оценка с учетом

$$
e^{C_{6}|\alpha|}\left(C_{6}|\alpha| e\right)^{\text {Card } U \cdot M}=o\left(M^{\varepsilon M}\right) \quad \text { при } \quad M \rightarrow \infty
$$

дает (2.15). 


\section{§ 3. Числовые приближающие формы}

В настоящем параграфе осушествляется традиционньй для метода ЗигеляШидловского переход от построенных функциональных к числовым приближаюшим формам. Однако, для этих целей используется новая схема рассуждений.

Ранг числовых приближающих форм. С каждым $\bar{s} \in \Theta$ удобно связать функцию $\mathscr{I}_{\bar{s}}: U \rightarrow \Omega$, действующую по правилу

$$
\mathscr{I}_{\bar{s}}(\bar{u})=\bar{s}-u_{1} \bar{e}_{11}-u_{2} \bar{e}_{21}-\cdots-u_{m} \bar{e}_{m 1}, \quad \bar{u}=\left(u_{1}, u_{2}, \ldots, u_{m}\right) \in U
$$

При этом функция $\mathscr{I}_{\bar{s}}$ определена не обязательно для всех $\bar{u} \in U$, поскольку мультииндекс в правой части ее определения не всегда имеет неотрицательные координаты.

В новых обозначениях многочлены $R_{\bar{s}^{*}}^{[n]}(z), n \geqslant 0, \bar{s}^{*}=N\left(\bar{e}_{11}+\bar{e}_{21}+\cdots+\bar{e}_{m 1}\right)$, от функций $f_{11}(z), f_{21}(z), \ldots, f_{m 1}(z)$ перепишутся в виде

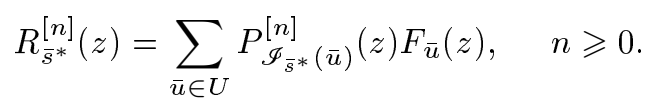

Положим

$$
\Omega^{*}=\bigcup_{\bar{u} \in U}\left\{\mathscr{I}_{\bar{s}^{*}}(\bar{u})\right\}, \quad \omega^{*}=\operatorname{Card} \Omega^{*}=\operatorname{Card} U .
$$

Важньм моментом метода Зигеля-Шидловского является доказательство того факта, что функциональный определитель, составленный из построенных форм, в нашем случае - это $\operatorname{det}\left(P_{\bar{\kappa}}^{[n]}(z)\right)_{n=0,1, \ldots, \omega-1 ; \bar{\kappa} \in \Omega}$, отличен от нуля. Именно такое утверждение было доказано Чудновским в статье [7] с помощью условия нормальности Зигеля для совокупности систем (0.6). На самом деле, в числовых приложениях нам понадобятся только формы (3.1). Поэтому можно воспользоваться более слабым аналогом указанного утверждения, накладывая менее жесткие (по сравнению с условием нормальности) ограничения (см. по этому поводу замечание к теореме III).

ПРЕДЛОЖЕНИЕ 3.1. Пусть система линейных однородных дифференциальньг уравнений (0.5) лежст в классе $\mathbf{W}^{0}(\alpha)$, функиии (0.11) линейно независимь над $\mathbb{C}(z)$ и $M$ достаточно велико, $M>M^{\prime}$. Тогда ранг числовой матриць

$$
\left(P_{\bar{\kappa}}^{[n]}(\alpha)\right)_{n=0,1, \ldots, \omega+\left[C_{7} \varepsilon M\right] ; \bar{\kappa} \in \Omega^{*}}
$$

в точности равен $\omega^{*}$.

ДокАЗАТЕльство. Допустим, что ранг совокупностилинейных форм $R^{[n]}(z ; \bar{a})$, $n=0,1,2, \ldots$, равен $\widetilde{\omega}, \widetilde{\omega} \leqslant \omega$. При этом $\widetilde{\omega} \geqslant 1$, поскольку $R^{[0]}(z ; \bar{a}) \not \equiv 0$ (см. по этому поводу замечание 2 к лемме 2.1 ).

Для произвольного решения

$$
a_{i j}=a_{i j}(z), \quad j=1, \ldots, m_{i}, \quad i=1, \ldots, m,
$$


системы (0.6) набор функций

$$
x_{\bar{\kappa}}(z)=\bar{a}^{\bar{\kappa}}(z) \prod_{i=1}^{m}\left(a_{i 1}(z) f_{i 1}(z)+\cdots+a_{i m_{i}}(z) f_{i m_{i}}(z)\right)^{N-u_{i}}, \quad \bar{\kappa} \in \Omega_{\bar{u}}, \quad \bar{u} \in U
$$

составляет решение системы линейных однородных дифференциальных уравнений

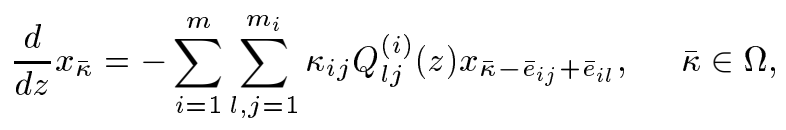

порядка $\omega$. Как следует из леммы 7 [1, гл. $3, \S 4]$, сушествует такая фундаментальная матрица решений $\left(x_{\bar{\kappa}, \eta}(z)\right)_{\bar{\kappa} \in \Omega ; \eta=1, \ldots, \omega}$ системы $(3.2)$, что если обозначить

$$
R_{\eta}^{[n]}(z)=\sum_{\bar{\kappa} \in \Omega} P_{\bar{\kappa}}^{[n]}(z) x_{\bar{\kappa}, \eta}(z), \quad n \geqslant 0, \quad \eta=1, \ldots, \omega
$$

TO

$$
R_{\eta}^{[n]}(z) \equiv 0, \quad n \geqslant 0, \quad \eta=\widetilde{\omega}+1, \ldots, \omega .
$$

Положим, следуя обозначениям $\S 1$,

$$
\begin{gathered}
\Lambda(z)=\operatorname{det}\left(x_{\bar{\kappa}, \eta}\right)_{\bar{\kappa} \in \Omega ; \eta=1, \ldots, \omega} \\
\lambda(\widetilde{\Omega} ; z)=\operatorname{det}\left(x_{\bar{\kappa}^{\prime}, \eta}\right)_{\bar{\kappa}^{\prime} \in \Omega \backslash \widetilde{\Omega} ; \eta=\widetilde{\omega}+1, \ldots, \omega}, \quad \widetilde{\Omega} \subset \Omega, \quad \operatorname{Card} \widetilde{\Omega}=\widetilde{\omega}
\end{gathered}
$$

(считаем $\lambda(\Omega ; z)=1$ в случае $\widetilde{\omega}=\omega)$.

Лемма 3.2. В случае $M>M^{\prime}$ существует множество $\widetilde{\Omega} \subset \Omega, \operatorname{Card} \widetilde{\Omega}=\widetilde{\omega}$, содержащее $\Omega^{*}$, такое, что $\lambda(\widetilde{\Omega} ; \alpha) \neq 0$.

ДоКАЗАТЕЛЬСтво. Если $\widetilde{\omega}=\omega$, то достаточно положить $\widetilde{\Omega}=\Omega$. Поэтому интересным представляется только случай $\widetilde{\omega}<\omega$.

Пусть утверждение леммы неверно и $\lambda(\widetilde{\Omega} ; \alpha)=0$ для любого $\widetilde{\Omega} \subset \Omega, \widetilde{\Omega} \supset \Omega^{*}$, $\operatorname{Card} \widetilde{\Omega}=\widetilde{\omega}$. Это означает, что ранг числовой матрицы

$$
\left(x_{\bar{\kappa}, \eta}(\alpha)\right)_{\bar{\kappa} \in \Omega \backslash \Omega^{*} ; \eta=\widetilde{\omega}+1, \ldots, \omega}
$$

меньше $\omega-\widetilde{\omega}$ и существует такая нетривиальная линейная числовая комбинация $\left(x_{\bar{\kappa}}^{*}(z)\right)_{\bar{\kappa} \in \Omega}$ столбцов матрицы

$$
\left(x_{\bar{\kappa}, \eta}(z)\right)_{\bar{\kappa} \in \Omega ; \eta=\widetilde{\omega}+1, \ldots, \omega},
$$

чTO

$$
x_{\bar{\kappa}}^{*}(\alpha)=0, \quad \bar{\kappa} \in \Omega \backslash \Omega^{*} .
$$


Таким образом, столбец $\left(x_{\bar{\kappa}}^{*}(z)\right)_{\bar{\kappa} \in \Omega}$ является нетривиальным решением системы (3.2), причем согласно (3.3) вьполнено

$$
\sum_{\bar{\kappa} \in \Omega} P_{\bar{\kappa}}^{[n]}(z) x_{\bar{\kappa}}^{*}(z) \equiv 0, \quad n \geqslant 0 .
$$

Пространство решений системы (3.2), удовлетворяюшее условиям (3.4), имеет размерность $\omega^{*}$. Ту же размерность имеет пространство, порожденное решениями

$$
x_{\bar{\kappa}}(z)=A_{\bar{u}} \bar{\varphi}^{\bar{\kappa}}(z), \quad A_{\bar{u}} \in \mathbb{C}, \quad \bar{\kappa} \in \Omega_{\bar{u}}, \quad \bar{u} \in U,
$$

системы (3.2), где участвующие функции (0.7) удовлетворяют системе (0.6) и условиям (0.8). В то же время решения вида (3.6) удовлетворяют условиям (3.4). Поэтому верно и обратное. Таким образом, полученное решение $\left(x_{\bar{\kappa}}^{*}(z)\right)_{\bar{\kappa} \in \Omega}$ представимо в виде

$$
x_{\bar{\kappa}}^{*}(z)=A_{\bar{u}} \bar{\varphi}^{\bar{\kappa}}(z), \quad \bar{\kappa} \in \Omega_{\bar{u}}, \quad \bar{u} \in U,
$$

с некоторыми фиксированными постоянными $A_{\bar{u}} \in \mathbb{C}$.

Поскольку решение (3.7) системы (3.2) нетривиально, $A_{\bar{u}^{\prime}} \neq 0$ для некоторого $\bar{u}^{\prime} \in U$. С другой стороны,

$$
\sum_{\bar{u} \in U} A_{\bar{u}} \sum_{\bar{\kappa} \in \Omega_{\bar{u}}} P_{\bar{\kappa}}^{[n]}(z) \bar{\varphi}^{\bar{\kappa}}(z) \equiv 0, \quad n \geqslant 0,
$$

согласно (3.5), откуда

$$
P_{\bar{\kappa}}^{[n]}(z) \equiv 0, \quad n \geqslant 0, \quad \bar{\kappa} \in \Omega_{\bar{u}^{\prime}},
$$

ввиду однородной алгебраической независимости функций $(0.7)$ над $\mathbb{C}(z)$.

Так как $\widetilde{\omega} \geqslant 1$, сушествуют мультииндексы $\bar{s} \in \Theta$ такие, что среди многочленов

$$
P_{\bar{s}-\bar{r}}^{[n]}(z), \quad n \geqslant 0, \quad|\bar{r}|=u_{i}, \quad i=1, \ldots, m,
$$

есть ненулевые. Выберем среди таких $\bar{s}$ мультииндекс $\bar{s}^{\prime}$ с наибольшей суммой $s_{11}^{\prime}+s_{21}^{\prime}+\cdots+s_{m 1}^{\prime}$. Тогда в каждой форме

$$
\begin{aligned}
R_{\bar{s}^{\prime}}^{[n]}(z) & =\sum_{\bar{u} \in U} \sum_{\substack{\bar{r}=\left(\bar{r}_{1}, \ldots, \bar{r}_{m}\right) \\
\bar{r}_{i} \mid=u_{i}, i=1, \ldots, m}} \prod_{i=1}^{m} \frac{u_{i} !}{r_{i 1} ! \cdots r_{i m_{i}} !} P_{\bar{s}^{\prime}-\bar{r}}^{[n]}(z) \bar{f}^{\bar{r}}(z) \\
& =\sum_{\bar{u} \in U} P_{\left.\bar{I}_{\bar{s}^{\prime}}(n]\right)}^{[n]}(z) f_{11}^{u_{1}}(z) f_{21}^{u_{2}}(z) \cdots f_{m 1}^{u_{m}}(z), \quad n \geqslant 0,
\end{aligned}
$$

участвует не более $\omega^{*}$ многочленов. Через $\widetilde{\omega}^{\prime}$ обозначим ранг совокупности форм (3.9) над $\mathbb{C}(z)$. Ввиду выбора $\bar{s}^{\prime}$ имеем $\widetilde{\omega}^{\prime} \geqslant 1$, а согласно (3.8) $\widetilde{\omega}^{\prime}<\omega^{*}$. Таким образом, для некоторого непустого подмножества

$$
\widetilde{\Omega}^{\prime} \subset \Omega^{\prime}=\bigcup_{\bar{u} \in U}\left\{\mathscr{I}_{\bar{s}^{\prime}}(\bar{u})\right\}, \quad \operatorname{Card} \widetilde{\Omega}^{\prime}=\widetilde{\omega}^{\prime},
$$


и рациональных функций

$$
D_{\bar{\kappa}, \bar{\kappa}^{\prime}}(z), \quad \bar{\kappa} \in \widetilde{\Omega}^{\prime}, \quad \bar{\kappa}^{\prime} \in \Omega^{\prime} \backslash \widetilde{\Omega}^{\prime},
$$

вьполняются равенства

$$
P_{\bar{\kappa}^{\prime}}^{[n]}(z)=\sum_{\bar{\kappa} \in \widetilde{\Omega}^{\prime}} P_{\bar{\kappa}}^{[n]}(z) D_{\bar{\kappa}, \bar{\kappa}^{\prime}}(z), \quad n \geqslant 0, \quad \bar{\kappa}^{\prime} \in \Omega^{\prime} \backslash \widetilde{\Omega}^{\prime} .
$$

Это означает, что существует $\widetilde{\Omega} \subset \Omega, \operatorname{Card} \widetilde{\Omega}=\widetilde{\omega}$, содержашее $\widetilde{\Omega}^{\prime}$, такое, что с рациональными функциями

$$
D_{\bar{\kappa}, \bar{\kappa}^{\prime}}(z), \quad \bar{\kappa} \in \widetilde{\Omega}, \quad \bar{\kappa}^{\prime} \in \Omega \backslash \widetilde{\Omega},
$$

справедливы равенства

$$
R_{\bar{\kappa}^{\prime}}^{[n]}(z)=\sum_{\bar{\kappa} \in \widetilde{\Omega}} P_{\bar{\kappa}}^{[n]}(z) D_{\bar{\kappa}, \bar{\kappa}^{\prime}}(z), \quad n \geqslant 0, \quad \bar{\kappa}^{\prime} \in \Omega \backslash \widetilde{\Omega}
$$

при этом

$$
D_{\bar{\kappa}, \bar{\kappa}^{\prime}}=0, \quad \bar{\kappa} \in \widetilde{\Omega} \backslash \widetilde{\Omega}^{\prime}, \quad \bar{\kappa}^{\prime} \in \Omega^{\prime} \backslash \widetilde{\Omega}^{\prime} .
$$

Рассуждая также, как и в работе $[8, \S 3]$ при доказательстве леммы 3.3, с помощью аналога леммы 3.1 и леммы 3.2 можно показать, что для рациональных функций (3.11) существует представление

$$
\begin{gathered}
D_{\bar{\kappa}, \bar{\kappa}^{\prime}}(z)=\frac{B_{\bar{\kappa}, \bar{\kappa}^{\prime}}(z)}{B(z)}, \quad B, B_{\bar{\kappa}, \bar{\kappa}^{\prime}} \in \mathbb{C}[z], \quad B \not \equiv 0, \\
\operatorname{deg} B \leqslant C_{8} \omega N, \quad \operatorname{deg} B_{\bar{\kappa}, \bar{\kappa}^{\prime}} \leqslant C_{8} \omega N, \quad \bar{\kappa} \in \widetilde{\Omega}, \quad \bar{\kappa}^{\prime} \in \Omega \backslash \widetilde{\Omega},
\end{gathered}
$$

где постоянная $C_{8}$ зависит только от совокупности функций $(0.9)$.

Таким образом, для некоторого множества $\mathscr{N} \subset\{0,1, \ldots, \widetilde{\omega}-1\}, \operatorname{Card} \mathscr{N}=\widetilde{\omega}^{\prime}$, выполнено

$$
\Delta(z)=\operatorname{det}\left(P_{\bar{\kappa}}^{[n]}(z)\right)_{n \in \mathcal{N} ; \bar{\kappa} \in \widetilde{\Omega}^{\prime}} \not \equiv 0,
$$

и согласно оценкам (2.10) находим, что

$$
\operatorname{deg} \Delta<\widetilde{\omega}^{\prime} M+\omega^{2} t .
$$

Из представления (3.12) и равенств (3.10) следует, что

$$
\begin{aligned}
B(z) R_{\bar{s}^{\prime}}^{[n]}(z) & =\sum_{\bar{\kappa} \in \widetilde{\Omega}^{\prime}} P_{\bar{\kappa}}^{[n]}(z) B(z) \bar{f}^{\bar{s}^{\prime}-\bar{\kappa}}(z)+\sum_{\bar{\kappa}^{\prime} \in \Omega^{\prime} \backslash \widetilde{\Omega}^{\prime}} P_{\bar{\kappa}^{\prime}}^{[n]}(z) B(z) \bar{f}^{\bar{s}^{\prime}-\bar{\kappa}^{\prime}}(z) \\
& =\sum_{\bar{\kappa} \in \widetilde{\Omega}^{\prime}} P_{\bar{\kappa}}^{[n]}(z)\left(B(z) \bar{f}^{\bar{s}^{\prime}-\bar{\kappa}}(z)+\sum_{\bar{\kappa}^{\prime} \in \Omega^{\prime} \backslash \widetilde{\Omega}^{\prime}} B_{\bar{\kappa}, \bar{\kappa}^{\prime}}(z) \bar{f}^{\bar{s}^{\prime}-\bar{\kappa}^{\prime}}(z)\right) \\
& =\sum_{\bar{\kappa} \in \widetilde{\Omega}^{\prime}} P_{\bar{\kappa}}^{[n]}(z) \widetilde{x}_{\bar{\kappa}}(z), \quad n \in \mathscr{N},
\end{aligned}
$$


где функции $\widetilde{x}_{\bar{\kappa}} \in \mathbb{C}\left[z, f_{11}, f_{21}, \ldots, f_{m 1}\right], \bar{\kappa} \in \widetilde{\Omega}^{\prime}$, имеют степень не выше $C_{8} \omega N$ по $z$ и степень $d$ по групше $f_{11}, f_{21}, \ldots, f_{m 1}$. Кроме того, $\widetilde{x}_{\bar{\kappa}} \neq \equiv 0, \bar{\kappa} \in \widetilde{\Omega}^{\prime}$, поскольку $B(z) \not \equiv 0$, а функции $(0.11)$, входящие в формы $\widetilde{x}_{\bar{\kappa}}, \bar{\kappa} \in \widetilde{\Omega}^{\prime}$, линейно независимы над $\mathbb{C}(z)$. По теореме 1 [9] (и замечанию к ней на случай алгебраической зависимости функций (0.9)) порядок нуля каждой из функций $\widetilde{x}_{\bar{\kappa}}(z)$ в точке $z=0$ не превосходит

$$
C_{9}\left(C_{8} \omega N+1\right) d^{m_{1}+\cdots+m_{m}}<C_{10} \omega N
$$

По построению множество $\widetilde{\Omega}^{\prime}$ пересекается с каждьм множеством $\Omega_{\bar{u}}, \bar{u} \in U$, не более чем по одному элементу. Положим

$$
U^{\prime}=\left\{\bar{u}: \widetilde{\Omega}^{\prime} \cap \Omega_{\bar{u}} \neq \varnothing\right\} \subset U, \quad \operatorname{Card} U^{\prime}=\widetilde{\omega}^{\prime}
$$

Пусть $\bar{u}^{*} \in U$ таково, что $M=M_{\bar{u}^{*}}$. Если $\bar{u}^{*} \in U^{\prime}$, то положим $\bar{r}=\widetilde{\Omega}^{\prime} \cap \Omega_{\bar{u}^{*}, \text { в }}$ противном случае считаем $\bar{r}$ некоторым элементом множества $\widetilde{\Omega}^{\prime}$. В обоих случаях множество

$$
U^{\prime \prime}=\left\{\bar{u}: \widetilde{\Omega}^{\prime} \backslash\{\bar{r}\} \cap \Omega_{\bar{u}} \neq \varnothing\right\} \subset U^{\prime}, \quad \operatorname{Card} U^{\prime \prime}=\operatorname{Card} U^{\prime}-1=\widetilde{\omega}^{\prime}-1
$$

не содержит $\bar{u}^{*}$.

Умножим теперь матрицу

$$
\left(P_{\bar{\kappa}}^{[n]}(z)\right)_{n \in \mathcal{N} ; \bar{\kappa} \in \widetilde{\Omega}^{\prime}}
$$

определитель которой равен $\Delta(z)$, справа на матрицу

$$
\left(\widetilde{x}_{\bar{\kappa}}(z) \mid \delta_{\bar{\kappa}, \bar{\kappa}^{\prime}}\right)_{\bar{\kappa} \in \widetilde{\Omega}^{\prime} ; \bar{\kappa}^{\prime} \in \widetilde{\Omega}^{\prime} \backslash\{\bar{r}\}}
$$

определитель которой равен $\widetilde{x}_{\bar{r}}$. Полученная в результате этого умножения матрица

$$
\left(B(z) R_{\bar{s}^{\prime}}^{[n]}(z) \mid P_{\bar{\kappa}^{\prime}}^{[n]}(z)\right)_{n \in \mathscr{N} ; \bar{\kappa}^{\prime} \in \widetilde{\Omega}^{\prime} \backslash\{\bar{r}\}}
$$

согласно (3.14) будет иметь ненулевой определитель $\Delta(z) \widetilde{x}_{\bar{r}}(z)$. В первом столбце этой матрицы стоят функциональные формы, порядок нуля которых в точке $z=0$ в соответствии с оценками $(2.12)$ не ниже $K-\widetilde{\omega}$; порядок нуля в точке $z=0$ многочленов $P_{\bar{\kappa}^{\prime}}^{[n]}(z), n \in \mathcal{N}$, согласно $(2.11)$ не ниже $M-M_{\bar{u}}-\widetilde{\omega}, \bar{\kappa}^{\prime} \in \Omega_{\bar{u}}$, $\bar{\kappa}^{\prime} \in \widetilde{\Omega}^{\prime} \backslash\{\bar{r}\}$. Поэтому

$$
\operatorname{ord}_{z=0} \Delta \widetilde{x}_{\bar{r}} \geqslant K+\sum_{\bar{u} \in U^{\prime \prime}}\left(M-M_{\bar{u}}\right)-\widetilde{\omega}^{\prime} \cdot \widetilde{\omega}>K+\sum_{\bar{u} \in U^{\prime \prime}}\left(M-M_{\bar{u}}\right)-\omega^{2}
$$


откуда

$$
\begin{aligned}
\underset{z=0}{\operatorname{ord}} \Delta & >K+\sum_{\bar{u} \in U^{\prime \prime}}\left(M-M_{\bar{u}}\right)-\omega^{2}-\operatorname{ord}_{z=0} \widetilde{x}_{\bar{r}} \\
& >K+\sum_{\bar{u} \in U^{\prime \prime}}\left(M-M_{\bar{u}}\right)-\omega^{2}-C_{10} \omega N \\
& \geqslant \operatorname{Card} U^{\prime \prime} \cdot M+\sum_{\bar{u} \in U \backslash U^{\prime \prime}} M_{\bar{u}}-2 \varepsilon M-\omega^{2}-C_{10} \omega N \\
& =\widetilde{\omega}^{\prime} M+\sum_{\substack{\bar{u} \in U \backslash U^{\prime \prime} \\
\bar{u} \neq \bar{u}^{*}}} M_{\bar{u}}-2 \varepsilon M-\omega^{2}-C_{10} \omega N .
\end{aligned}
$$

Сопоставляя последнюю оценку с неравенством (3.13), заключаем, что

$$
\sum_{\substack{\bar{u} \in U \backslash U^{\prime \prime} \\ \bar{u} \neq \bar{u}^{*}}} M_{\bar{u}}<2 \varepsilon M+\omega^{2}(t+1)+C_{10} \omega N<3 \varepsilon M
$$

для всех $M>M^{\prime}$. Суммирование в левой части последнего неравенства происходит по непустому множеству, поскольку $\operatorname{Card} U^{\prime \prime}=\widetilde{\omega}^{\prime}-1 \leqslant \omega^{*}-2=\operatorname{Card} U-2$. $\mathrm{C}$ другой стороны, $M_{\bar{u}} \geqslant 3 \varepsilon M$ для всех $\bar{u} \in U$ согласно определению параметров построения, что противоречит последнему неравенству.

Тем самым, исходное предположение является ложным, а следовательно, выполнено утверждение леммы 3.2 .

Для выбранного в соответствии с леммой 3.2 множества $\widetilde{\Omega} \subset \Omega$, Card $\widetilde{\Omega}=\widetilde{\omega}$, обозначим $\Delta(z)=\operatorname{det}\left(P_{\bar{\kappa}}^{[n]}(z)\right)_{n=0,1, \ldots, \widetilde{\omega}-1 ; \bar{\kappa} \in \widetilde{\Omega}}$. По лемме $1.3 \Delta(z) \not \equiv 0$. Кроме того, если в матрице, соответствующей определителю $\Delta(z)$, некоторый столбец заменить с помощью невырожденного линейного преобразования на столбец $\left(R_{\bar{s}^{*}}^{[n]}(z)\right)_{n=0,1, \ldots, \tilde{\omega}-1}$, то согласно оценкам (2.12) мы получим, что

$$
\operatorname{ord}_{z=0} \Delta(z)>K-\widetilde{\omega} \geqslant M
$$

Подставляя оценки $(2.10),(2.13)$ и (3.15) в лемму 1.6, получим, что

$$
\begin{aligned}
\underset{z=\alpha}{\operatorname{ord} \Delta(z)} & <\frac{2 \widetilde{\omega}}{\log M}\left(\log \left(\left(C_{1} N\right) \frac{\widetilde{\omega}}{(M-1) !} C_{0}^{M / \varepsilon}\right)+2(M+t \widetilde{\omega}-1)(1+\log \widetilde{\omega})\right) \\
& \leqslant \frac{2 \omega}{\log M}\left(\omega \log \left(C_{1} N\right)+t \omega \log (2 M)+\frac{M}{\varepsilon} \log C_{0}+2(M+t \omega)(1+\log \omega)\right) \\
& \leqslant \frac{C_{11} \omega M}{\varepsilon \log M} \leqslant C_{7} \varepsilon M,
\end{aligned}
$$

поскольку

$$
\frac{\omega}{\varepsilon \log M} \asymp \varepsilon
$$

в силу выбора (2.2).

Для завершения доказательства предложения 3.1 остается подставить оценку (3.16) в лемму 1.3 и воспользоваться тем, что $\Omega^{*} \subset \widetilde{\Omega}$. 
Арифметические свойства числовых форм. В этом пункте мы подытожим полученные результаты и, следуя работе А. Бейкера [3], осушествим доказательство теоремы II.

ПРЕДЛОЖЕНИЕ 3.3. Пусть система линейнъх однородных дифференциальных уравнений (0.5) лежит в классе $\mathbf{W}^{0}(\alpha)$, функиии $(0.11)$ линейно независимьи над $\mathbb{C}(z)$ и $M>M^{\prime}$. Тогда существуют иелье числа $\rho_{\bar{u}}^{[n]}$, удовлетворяющие оценкам

$$
\left|\rho_{\bar{u}}^{[n]}\right|<M^{M_{\bar{u}}+C_{12} \varepsilon M}, \quad n=1, \ldots, \operatorname{Card} U, \quad \bar{u} \in U
$$

такие, что для числовых форм

$$
\xi^{[n]}=\sum_{\bar{u} \in U} \rho_{\bar{u}}^{[n]} F_{\bar{u}}(\alpha)=\sum_{\bar{u} \in U} \rho_{\bar{u}}^{[n]} f_{11}^{u_{1}}(\alpha) f_{21}^{u_{2}}(\alpha) \cdots f_{m 1}^{u_{m}}(\alpha), \quad n=1, \ldots, \text { Card } U
$$

справедливьь оценки

$$
\left|\xi^{[n]}\right|<M^{-\sum_{\bar{u} \in U} M_{\bar{u}}+M+C_{13} \varepsilon M}, \quad n=1, \ldots, \operatorname{Card} U
$$

u, кроме того,

$$
\operatorname{det}\left(\rho_{\bar{u}}^{[n]}\right)_{n=1, \ldots, \operatorname{Card} U ; \bar{u} \in U} \neq 0
$$

ДокАЗАТЕЛьство. Воспользуемся предложением 3.1: сушествуют целые числа $\nu_{1}, \ldots, \nu_{\omega^{*}}, 0 \leqslant \nu_{1}<\cdots<\nu_{\omega^{*}} \leqslant \omega+\left[C_{7} \varepsilon M\right]<C_{2} \varepsilon M, \omega^{*}=\operatorname{Card} U$, такие, что

$$
\operatorname{det}\left(P_{\bar{\kappa}}^{\left[\nu_{n}\right]}(\alpha)\right)_{n=1, \ldots, \omega^{*} ; \bar{\kappa} \in \Omega^{*}} \neq 0
$$

Пусть $\alpha=a / b$, где $a \in \mathbb{Z}, b \in \mathbb{N}$. Для целых чисел

$$
\rho_{\bar{u}}^{[n]}=b^{M+t \nu_{n}}\left(M+t \nu_{n}\right) ! P_{\mathscr{I}_{\bar{s}^{*}}(\bar{u})}^{[n]}(\alpha), \quad n=1, \ldots, \omega^{*}, \quad \bar{u} \in U,
$$

согласно оценке (2.14) имеем

$$
\begin{aligned}
\left|\rho_{\bar{u}}^{[n]}\right| & <b^{M+t \nu_{n}}\left(M+t \nu_{n}\right) ! \sum_{\mu=\max \left\{0, M-M_{\bar{u}}-\nu_{n}\right\}}^{M+t \nu_{n}-1} \frac{M^{C_{3} \varepsilon M}}{\mu !}|\alpha|^{\mu} \\
& <b^{M+t C_{2} \varepsilon M}|a|^{M} e^{|\alpha|}(2 e)^{M}(2 M)^{(t+1) C_{2} \varepsilon M} M^{M_{\bar{u}}} \\
& \leqslant M^{M_{\bar{u}}+C_{12} \varepsilon M}, \quad n=1, \ldots, \omega^{*}, \quad \bar{u} \in U,
\end{aligned}
$$


поскольку

$$
\begin{gathered}
\sum_{\mu=\max \left\{0, M-M_{\bar{u}}-\nu\right\}} \frac{M^{C_{3} \varepsilon M}}{\mu !}|\alpha|^{\mu}<\frac{|\alpha| \max \left\{0, M-M_{\bar{u}}-\nu\right\}}{\max \left\{0, M-M_{\bar{u}}-\nu\right\} !} \sum_{\mu=0}^{\infty} \frac{|\alpha|^{\mu}}{\mu !} \\
\quad \leqslant \frac{|a|^{M}}{\max \left\{0, M-M_{\bar{u}}-\nu\right\} !} \cdot e^{|\alpha|}, \\
\frac{(M+t \nu) !}{\max \left\{0, M-M_{\bar{u}}-\nu\right\} !}<\frac{(2 M)^{M+t \nu+\nu}}{(M-t \nu+\nu) !}<\frac{(2 M)^{M+t \nu+\nu}}{\left(M-M_{\bar{u}}\right)^{M-M_{\bar{u}}} \cdot e^{-\left(M-M_{\bar{u}}\right)}} \\
<\frac{M^{M-M_{\bar{u}} \cdot e^{-M}}=(2 e)^{M}(2 M)^{(t+1) \nu} M^{M_{\bar{u}}},}{\nu<\frac{M}{t+1}, \quad \bar{u} \in U .}
\end{gathered}
$$

В последней оценке мы применили неравенство

$$
\frac{M^{M-M_{\bar{u}}}}{\left(M-M_{\bar{u}}\right)^{M-M_{\bar{u}}}}=\left(1+\frac{M_{\bar{u}}}{M-M_{\bar{u}}}\right)^{M-M_{\bar{u}}}<e^{M_{\bar{u}}}, \quad \bar{u} \in U .
$$

Теперь воспользуемся оценкой (2.15) для полученных форм (3.18):

$$
\begin{aligned}
\left|\xi^{[n]}\right| & =b^{M+t \nu_{n}}\left(M+t \nu_{n}\right) !\left|R_{\bar{s}^{*}}^{\left[\nu_{n}\right]}(\alpha)\right|<b^{M+t C_{2} \varepsilon M}(2 M)^{M+t C_{2} \varepsilon M^{M}} M^{C_{4} \varepsilon M-K} \\
& \leqslant M^{-\sum_{\bar{u} \in U} M_{\bar{u}}+M+C_{13} \varepsilon M}, \quad n=1, \ldots, \omega^{*} .
\end{aligned}
$$

Предложение доказано.

ДОКАЗАТЕЛЬСТВо ТЕОРЕМЫ II. ПоЛОжим

$$
\begin{gathered}
C_{14}=\frac{1}{2} \min _{\bar{u} \in U}\left\{\left|F_{\bar{u}}(\alpha)\right|\right\} \\
C_{15}=\max \left\{3,\left(\omega^{*}-2\right) C_{12}+C_{13}+\frac{1}{\varepsilon\left(M^{\prime}\right) \cdot M^{\prime} \log M^{\prime}} \log \frac{\omega^{*} !}{C_{14}}\right\},
\end{gathered}
$$

где постоянные $M^{\prime}, C_{12}, C_{13}$ определены в предложении $3.3, \omega^{*}=\operatorname{Card} U$ и

$$
\varepsilon=\varepsilon(M) \asymp(\log M)^{-1 /\left(m_{1}+\cdots+m_{m}-m+2\right)}
$$

определяется из равенств (2.2). Тогда при всех $M>M^{\prime}$ будут справедливы неравенства $C_{15} \geqslant 3$ и

$$
\omega^{*} ! M^{\left(\omega^{*}-2\right) C_{12} \varepsilon M+C_{13} \varepsilon M-C_{15} \varepsilon M} \leqslant C_{14}
$$

Для заданной числовой формы

$$
r=\sum_{\bar{u} \in U} h_{\bar{u}} F_{\bar{u}}(\alpha), \quad h_{\bar{u}} \in \mathbb{Z} \backslash\{0\}, \quad \bar{u} \in U,
$$


обозначим через $M$ наименьшее целое число, удовлетворяющее неравенству

$$
M^{\left(1-C_{15} \varepsilon\right) M} \geqslant H=\max _{\bar{u} \in U}\left\{\left|h_{\bar{u}}\right|\right\} \geqslant 3 .
$$

Тогда для всех $M>M^{\prime \prime}$ выполнено $H>M^{M / 2}$ и, в частности,

$$
\log \log H>\log M+\log \log M-\log 2>\log M
$$

откуда и из (3.21)

$$
\varkappa=\varkappa(H)=(\log \log H)^{-1 /\left(m_{1}+\cdots+m_{m}-m+2\right)}>C_{16} \varepsilon .
$$

Следовательно, при $M>M^{\prime \prime}$ справедлива оценка

$$
M^{\varepsilon M}<H^{2 \varepsilon}<H^{2 \varkappa / C_{16}}
$$

Возьмем $\bar{u}^{*} \in U$ таким образом, что $H=\left|h_{\bar{u}^{*}}\right|$, и положим $M_{\bar{u}^{*}}=M$. Целые числа $M_{\bar{u}}, \bar{u} \neq \bar{u}^{*}$, выберем так, чтобы они удовлетворяли неравенствам

$$
M_{\bar{u}} \geqslant \frac{\log \left|h_{\bar{u}}\right|}{\log M}+C_{15} \varepsilon M>M_{\bar{u}}-1, \quad \bar{u} \in U \backslash\left\{\bar{u}^{*}\right\}
$$

откуда, в частности,

$$
M_{\bar{u}} \geqslant \frac{\log \left|h_{\bar{u}}\right|}{\log M}+C_{15} \varepsilon M \geqslant C_{15} \varepsilon M \geqslant 3 \varepsilon M, \quad \bar{u} \in U \backslash\left\{\bar{u}^{*}\right\} .
$$

Из условий (3.24) и (3.26) следует, что

$$
M \geqslant \frac{\log H}{\log M}+C_{15} \varepsilon M \geqslant \frac{\log \left|h_{\bar{u}}\right|}{\log M}+C_{15} \varepsilon M>M_{\bar{u}}-1, \quad \bar{u} \in U \backslash\left\{\bar{u}^{*}\right\},
$$

откуда

$$
M=M_{\bar{u}^{*}}=\max _{\bar{u} \in U}\left\{M_{\bar{u}}\right\}
$$

Условия (3.27) и (3.28) обеспечивают выбор (2.2). Кроме того, можно считать $M>M^{\prime}$ и $M>M^{\prime \prime}$, поскольку форм (3.23), для которых $M \leqslant \max \left\{M^{\prime}, M^{\prime \prime}\right\}$, только конечное количество, а значит, для них верна оценка теоремы. Итак, можно воспользоваться предложением 3.3. Формы (3.18) согласно условию (3.20) линейно независимы. Поэтому среди них можно выбрать $m-1$ форму, для определенности $\xi^{[2]}, \ldots, \xi^{\left[\omega^{*}\right]}$, так, что формы $r, \xi^{[2]}, \ldots, \xi^{\left[\omega^{*}\right]}$ будут линейно независимы. Из целых коэффициентов этих форм составим определитель

$$
\tau=\operatorname{det}\left(\begin{array}{c}
h_{\bar{u}} \\
\rho_{\bar{u}}^{[2]} \\
\vdots \\
\rho_{\bar{u}}^{\left[\omega^{*}\right]}
\end{array}\right)_{\bar{u} \in U}
$$


отличньй от нуля ввиду линейной независимости форм (3.23) и (3.18). Поскольку $\tau \in \mathbb{Z}$, имеем $|\tau| \geqslant 1$. В матрице, соответствуюшей определителю $\tau$, к столбцу с номером $\bar{u}^{*}$, домноженному на $F_{\bar{u}^{*}}(\alpha)$, прибавим остальные столбцы с номерами $\bar{u} \in U \backslash\left\{\bar{u}^{*}\right\}$, домноженные на $F_{\bar{u}}(\alpha)$ соответственно. Определитель получившейся матрицы равен $\tau F_{\bar{u}^{*}}(\alpha)$, а с другой стороны, его можно представить в виде

$$
\tau_{1} r+\sum_{n=2}^{\omega^{*}} \tau_{n} \xi^{[n]},
$$

где $\tau_{n}$ - алгебраическое дополнение элемента $n$-й строки и столбца с номером $\bar{u}^{*}$ в $\tau$. Следовательно,

$$
\begin{aligned}
\left|\tau_{1}\right| \cdot|r| & =\left|\tau F_{\bar{u}^{*}}(\alpha)-\sum_{n=2}^{\omega^{*}} \tau_{n} \xi^{[n]}\right| \geqslant|\tau| \cdot\left|F_{\bar{u}^{*}}(\alpha)\right|-\sum_{n=2}^{\omega^{*}}\left|\tau_{n}\right| \cdot\left|\xi^{[n]}\right| \\
& \geqslant\left|F_{\bar{u}^{*}}(\alpha)\right|-\sum_{n=2}^{\omega^{*}}\left|\tau_{n}\right| \cdot\left|\xi^{[n]}\right| \geqslant 2 C_{14}-\sum_{n=2}^{\omega^{*}}\left|\tau_{n}\right| \cdot\left|\xi^{[n]}\right| .
\end{aligned}
$$

С помощью оценок (3.17) и поскольку

$$
\left|h_{\bar{u}}\right| \leqslant M^{M_{\bar{u}}-C_{15} \varepsilon M}, \quad \bar{u} \in U \backslash\left\{\bar{u}^{*}\right\},
$$

из (3.26), находим

$$
\begin{aligned}
& \left|\tau_{1}\right|<\left(\omega^{*}-1\right) ! \prod_{\bar{u} \neq \bar{u}^{*}} M^{M_{\bar{u}}+C_{12} \varepsilon M}, \\
& \left|\tau_{n}\right|<\left(\omega^{*}-1\right) ! \prod_{\bar{u} \neq \bar{u}^{*}} M^{M_{\bar{u}}} \cdot M^{\left(\omega^{*}-2\right) C_{12} \varepsilon M-C_{15} \varepsilon M}, \quad n=2, \ldots, \omega^{*},
\end{aligned}
$$

откуда согласно (3.19) и (3.22) имеем

$$
\left|\tau_{n}\right| \cdot\left|\xi^{[n]}\right|<\left(\omega^{*}-1\right) ! M^{\left(\omega^{*}-2\right) C_{12} \varepsilon M-C_{15} \varepsilon M+C_{13} \varepsilon M} \leqslant \frac{C_{14}}{\omega^{*}}, \quad n=2, \ldots, \omega^{*} .
$$

Подставляя последнее неравенство в (3.29), имеем

$$
\left|\tau_{1}\right| \cdot|r|>2 C_{14}-\left(\omega^{*}-1\right) \frac{C_{14}}{\omega^{*}}>C_{14}
$$

откуда, используя $(3.25),(3.26),(3.30)$, находим

$$
\begin{aligned}
|r|>C_{14}\left|\tau_{1}\right|^{-1} & >\frac{C_{14}}{\left(\omega^{*}-1\right) !} \prod_{\bar{u} \neq \bar{u}^{*}}\left(\left|h_{\bar{u}}\right| M^{C_{15} \varepsilon M+1+C_{12} \varepsilon M}\right)^{-1} \\
& >\frac{C_{14}}{\left(\omega^{*}-1\right) !} \prod_{\bar{u} \neq \bar{u}^{*}}\left|h_{\bar{u}}\right|^{-1} \cdot H^{-2\left(\omega^{*}-1\right)\left(C_{15}+1+C_{12}\right) \varkappa / C_{16}} .
\end{aligned}
$$

Последнее неравенство в точности означает оценку теоремы II с $C=C_{14} /\left(\omega^{*}-1\right)$ ! и $\gamma=2\left(\omega^{*}-1\right)\left(C_{15}+1+C_{12}\right) / C_{16}$. 


\section{Список литературы}

1. Шидловский А. Б. Трансцендентные числа. М.: Наука, 1987.

2. Спринджсук В. Г. Метрическая теория диофантовых приближений. М.: Наука, 1977.

3. Baker $A$. On some diophantine inequalities involving the exponential function // Canad. J. Math. 1965. V. 17. № 4. P. 616-626.

4. Siegel C. L. Über einige Anwendungen diophantischer Approximationen // Abh. Preuss. Wiss. Phys.-Math. Kl. 1929-1930. №1. P. 1-70.

5. Макаров Ю. Н. Об оценках меры линейной независимости значений $E$-фуннций // Вестн. МГУ. Сер. 1. Матем., мех. 1978. № 2. С. 3-12.

6. Lang S. Introduction to Transcendental Numbers. Reading: Addison Wesley Publishing Co., 1966.

7. Chudnovsky G. V. On some applications of diophantine approximations // Proc. Nat. Acad. Sci. USA. 1984. V. 81. March. P. 1926-1930.

8. Зудилин В. В. О рациональных приближениях значений одного класса целых функций // Матем. сб. 1995. Т. 186. № 4. С. 89-124.

9. Нестеренко Ю. В. Оценки числа нулей функций некоторых классов // Acta Arith. 1989. T. 53. № 1. C. 29-46.

Московский государственный университет им. М.В. Ломоносова

E-mail: wadim@ipsun.ras.ru
Поступила в редакцию 14.12.1995 